Citation:

Rodney A. Stewart, Khoi Nguyen, Cara Beal, Hong Zhang, Oz Sahin, Edoardo Bertone, Abel Silva Vieira, Andrea Castelletti, Andrea Cominola, Matteo Giuliani, Damien Giurco, Michael Blumenstein, Andrea Turner, Ariane Liu, Steven Kenway, Dragan A. Savić, Christos Makropoulos, Panagiotis Kossieris, Integrated intelligent water-energy metering systems and informatics:

Visioning a digital multi-utility service provider, Environmental Modelling \& Software, Volume 105, July 2018, Pages 94-117, ISSN 1364-8152,

https://doi.org/10.1016/j.envsoft . 2018.03.006.

\title{
Integrated intelligent water-energy metering systems and informatics: Visioning a digital multi-utility service provider
}

\section{Authors and affiliations:}

Prof. Rodney A. Stewart ${ }^{*}, 1,2$, Dr. Khoi Nguyen ${ }^{1,2}$, Dr. Cara Beal ${ }^{1,2}$, Prof. Hong Zhang ${ }^{1,2}$, Dr. Oz Sahin $^{1,2}$, Dr. Edoardo Bertone ${ }^{1,2}$, Abel Silva Vieira ${ }^{1,2,3}$

Griffith School of Engineering ${ }^{1}$, Cities Research Institute ${ }^{2}$, Griffith University, Queensland, Australia ${ }^{3}$ City of Gold Coast, Queensland, Australia

*Corresponding author: r.stewart@griffith.edu.au

Phone: +61 755528778

Prof. Andrea Castelletti ${ }^{1}$, Dr. Andrea Cominola ${ }^{1}$, Dr. Matteo Giuliani ${ }^{1}$

Department of Electronics, Information and Bioengineering ${ }^{1}$, Politecnico di Milano, Milano, Italy Prof. Damien Giurco ${ }^{1}$, Prof. Michael Blumenstein ${ }^{2}$, Andrea Turner ${ }^{1}$, Dr Ariane Liu ${ }^{1}$

Institute for Sustainable Futures ${ }^{1}$, Faculty of Engineering and IT $^{2}$, University Technology Sydney, New South Wales, Australia

A/Prof. Steven Kenway ${ }^{1}$

School of Chemical Engineering ${ }^{1}$, University of Queensland, Queensland, Australia

Prof. Dragan A. Savić1, 2

${ }^{1}$ Co-Director, Centre for Water Systems, ${ }^{2}$ Director, EPSRC Centre for Doctoral Training in Water Informatics: Science and Engineering (WISE CDT) University of Exeter, United Kingdom

A/Prof. Christos Makropoulos ${ }^{1}$, Panagiotis Kossieris ${ }^{1}$

${ }^{1}$ School of Civil Engineering, National Technical University of Athens, Athens, Attiki, Greece.

\section{Position paper submission to:}

Journal: Environmental Modelling \& Software, Elsevier

Thematic Issue: Smart Systems for Urban Water Management 


\begin{abstract}
Advanced metering technologies coupled with informatics creates an opportunity to form digital multiutility service providers. These providers will be able to concurrently collect a customers' medium-high resolution water, electricity and gas demand data and provide user-friendly platforms to feed this information back to customers and supply/distribution utility organisations. Providers that can install low-cost integrative systems will reap the benefits of derived operational synergies and access to mass markets not bounded by historical city, state or country limits. This paper provides a vision of the required transformative process and features of an integrated multi-utility service provider covering the system architecture, opportunities and benefits, impediments and strategies, and business opportunities. The heart of the paper is focused on demonstrating data modelling processes and informatics opportunities for contemporaneously collected demand data, through illustrative examples and four informative water-energy nexus case studies. Finally, the paper provides an overview of the transformative $\mathrm{R} \& \mathrm{D}$ priorities to realise the vision.
\end{abstract}

Key words: Multi-utility; digital metering; smart metering; demand management; big data; waterenergy nexus.

\title{
1. Background
}

\subsection{Digital multi-utility futures}

Imagine a future where a technology company is the retailer of water, wastewater, electricity and gas services, for your home or business. At first thought this concept seems confounding but in reality this future is not too far away, as integrated digital metering, advanced communications and big data analytics paves the way for the creation of a global multi-utility service provider catering for millions, if not billions, of customers.

Digital disruption has already transformed a number of other industries globally, but the utility sector has been slow to embrace digital transformation technologies. This is largely because of their conservative nature, often underpinned by a natural monopoly status that is government-owned or tightly regulated, thereby preventing the easy access of entrepreneurs' reinventing typical business supply chains (Kiesling, 2016; Tayal, 2016). But, for instance, the rapid rise of $U_{b e r}{ }^{T M}$ has shown us that even highly regulated and protected industries, such as the taxi industry, will inevitably be pressured to open up to innovative products offering unprecedented customer savings and value-adding services. 
This position paper will firstly provide a background and a vision for an integrated digital multi-utility service provider. The system architecture for such a provider will be discussed along with the opportunities and benefits, as well as impediments and challenges, for utility transition. The paper then hones in on its core objective, which is to demonstrate the opportunities and benefits of modelling concurrently collected and autonomously analysed water and energy data by presenting case studies and empirical data examples. The paper finishes with a discussion on the core research and development priorities to realise the vision of a digital multi-utility.

\subsection{Changing utility sector paradigm}

Traditional provision of water and energy (electricity or gas), until quite recently, was a conservative process whereby quasi-government owned utilities offered a unidimensional, one way service to their customers. As expectations to provide a greener, leaner and customer-focussed utility sharply rise, it has become clear that conventional means of water and energy provision are becoming outdated and will not be able to meet the requirements of the digital information age (Kabalci, 2016). By necessity in meeting these changing needs, utility meters are being transformed from simple measurement devices where manual collection of only 1 data point (i.e. consumption) via mechanical meters on a monthly or quarterly basis, to more complex and “intelligent” metering. In 1999, Marvin et al. termed such 'smart' meters as socio-technical systems where enhanced informational and communication capacities allowed for a deeper and dynamic understanding of both the supply and demand metabolism of the utility. Nearly 20 years on, there is now a wealth of literature documenting the paradigm shift toward the digital water and energy utility (e.g. Stewart et al. 2010; Depuru et al. 2011; Fang et al. 2012; Stewart et al. 2013; Gans et al. 2013; Beal and Flynn 2015; Cominola et al. 2015; Tuballa and Abundo, 2016; Pitì et al. 2017).

As the momentum gathers, there is increasing pressure on the utility sector to transition to the digital age. Tubulla and Abundo (2016) describe the breadth of energy utilities that are embracing disruptive technologies to improve the efficiency and customer service of their business - including Europe, North America Asia, and Australia. While the water sector has been slower to adopt such disruptive technologies, the impetus is growing, driven largely by customer expectations and increasingly expensive water operations. There is a growing realization by water utilities that the enormous opportunities digital metering provides need to be harnessed, and a broader systems and futures perspective used, to determine the extent and direction of those opportunities (Turner and White, 2017).

With digital metering comes many challenges, including capital costs, technology redundancy, business transformation, risk mitigation and customer expectations while maintaining billing equity. One of the main challenges, however, is likely to be how the vast volume of continuously accumulating information is used to ensure that digital technology enhances urban water, electricity and gas 
management. Addressing this 'big data' challenge through targeted modelling of concurrently collected utility data is the key focus of this paper.

\subsection{Advent of intelligent metering technologies}

A smart water or energy grid essentially refers to the integration and remote communication of information via enabling technologies such as sensors, meters, and automated controls that continuously and remotely monitor the water, electricity or gas distribution system. The advent and advancement of these innovative enabling technologies has allowed an almost endless capacity to monitor many different parameters. For water distribution this includes pressure, quality, flow rates, temperature and leaks. In energy distribution systems, peak load shifting, losses and theft, resource storage and time of day demand are all key features of a smart energy grid (Depuru et al. 2011; Rhodes et al. 2014).

Within a decade, technology has rapidly become more sophisticated, from needing separate hardware and software to collect, store, transfer and analyse a gigabyte of data, to now having one piece of technology that combines hardware, software and firmware to provide near-real time, tailored reports to utilities and customers. Selected examples of research studies presenting digital metering technology and its applications for managing water and energy distribution are provided in Table 1. 
Table 1. Selected examples of research on digital metering technology and applications for water and energy utilities

\begin{tabular}{|c|c|c|c|c|}
\hline Technology & Study aim / methods & Sector & Key points / outcomes & $\begin{array}{l}\text { Location and } \\
\text { source }\end{array}$ \\
\hline Digital water meters (1 hour interval) & $\begin{array}{l}\text { Develop models of water consumer behaviors and foster } \\
\text { water saving behaviours by raising consumer awareness. }\end{array}$ & Water & $\begin{array}{l}\text { Water consumers adopting the SmartH2O } \\
\text { digital platform achieved substantial water } \\
\text { savings, compared to those who did not adopt it } \\
\text { (control group). }\end{array}$ & $\begin{array}{l}\text { Spain, Switzerland } \\
\text { (Rizzoli et al., 2014) }\end{array}$ \\
\hline $\begin{array}{l}\text { Digital electricity and water meters, microcontroller } \\
\text { and Global Service Mobile (GSM) modem for } \\
\text { communication }\end{array}$ & $\begin{array}{l}\text { Test energy and water smart meters to see how this } \\
\text { technology can improve billing system. Technology } \\
\text { prototypes tested and assessed. }\end{array}$ & $\begin{array}{l}\text { Electricity } \\
\text { and water }\end{array}$ & $\begin{array}{l}\text { High accuracy results for billing compared with } \\
\text { existing systems. }\end{array}$ & $\begin{array}{l}\text { Sharjah, UAE (Al- } \\
\text { Rousan and Al-Ali, } \\
\text { 2006) }\end{array}$ \\
\hline $\begin{array}{l}\text { Digital water meter at resolution of } 0.014 \text { litre per } \\
\text { minute (L/min) and data loggers ( } 5 \text { second (s) } \\
\text { intervals). Data remotely transferred by email for } \\
\text { processing and analysis }\end{array}$ & $\begin{array}{l}\text { To generate baseline end-use or micro-component water } \\
\text { data from residential homes to inform targeted demand } \\
\text { management strategies. Householder survey combined } \\
\text { with big data. }\end{array}$ & Water & $\begin{array}{l}\text { Report showcasing the breadth of applications } \\
\text { of big data and benefits to utility and customers }\end{array}$ & $\begin{array}{l}\text { South east Qld, } \\
\text { Australia (Beal and } \\
\text { Stewart 2011) }\end{array}$ \\
\hline $\begin{array}{l}\text { Digital electricity meters with real-time customer use } \\
\text { displayed on visual display digital monitor ("keypad } \\
\text { meters"). }\end{array}$ & $\begin{array}{l}\text { To compare consumption before and after smart-meter } \\
\text { enabled feedback to customers. Modelling using } \\
\text { residential billing data. }\end{array}$ & Electricity & $\begin{array}{l}\text { Electricity consumption reduction was } \\
\text { calculated from post-installation of smart meter } \\
\text { with visual display. }\end{array}$ & $\begin{array}{l}\text { Northern Ireland (Gans } \\
\text { et al. 2013) }\end{array}$ \\
\hline Digital electricity meters (10 min intervals) & $\begin{array}{l}\text { Research on how data mining and analytics can reveal } \\
\text { relationships useful for customer and utilities for future } \\
\text { demand management. Householder survey combined } \\
\text { with big data. }\end{array}$ & Electricity & $\begin{array}{l}\text { Data informatics revealed relationships between } \\
\text { use and household stock, size, climate and } \\
\text { socio-demographics. }\end{array}$ & $\begin{array}{l}\text { California, USA } \\
\text { (Kavousian et al. 2013) }\end{array}$ \\
\hline $\begin{array}{l}\text { Digital water meters ( } 3 \mathrm{~L} / \mathrm{hr} \text {, every hour). Meters are } \\
\text { read via drive-by units. }\end{array}$ & $\begin{array}{l}\text { Meter data used to identify and classify leak typology } \\
\text { and the impact of leak notification to customer. }\end{array}$ & Water & $\begin{array}{l}\text { Study confirmed that smart metering provided } \\
\text { water utilities with a powerful tool for rapid } \\
\text { leak detection (and subsequent rectification). }\end{array}$ & $\begin{array}{l}\text { Hervey Bay, Australia } \\
\text { (Britton et al. 2013) }\end{array}$ \\
\hline $\begin{array}{l}\text { Digital water meters ( } 1 \text { gal resolution every } 15 \mathrm{~s}) \text {; } \\
\text { digital gas meters ( } 2 \text { cubic feet every } 15 \text { s); digital } \\
\text { electricity meters ( } 10 \text { watt resolution at } 1 \text { min to } 15 \\
\text { min interval) }\end{array}$ & $\begin{array}{l}\text { Number of integrated and controlled demonstration } \\
\text { projects aimed at testing large-scale smart grid } \\
\text { deployment. }\end{array}$ & $\begin{array}{l}\text { Gas, } \\
\text { electricity } \\
\text { and water }\end{array}$ & $\begin{array}{l}\text { Tested technologies and analyses of novel } \\
\text { datasets to identify potential for grid planning } \\
\text { and understanding how customers will interface } \\
\text { with new devices, information, and price } \\
\text { signals. }\end{array}$ & $\begin{array}{l}\text { Texas, USA (Rhodes et } \\
\text { al. 2014) }\end{array}$ \\
\hline Digital electricity meters (30 min intervals) & $\begin{array}{l}\text { Multiple regression analysis was used to determine } \\
\text { household characteristics e.g. the number of inhabitants, } \\
\text { the size of the property, and the number of appliances by } \\
\text { analyzing households' electricity consumption. }\end{array}$ & Electricity & $\begin{array}{l}\text { Data can be used to develop tailored demand } \\
\text { management messaging to customers, demand } \\
\text { forecasting profiles for utilities and insights for } \\
\text { policy makers around regulating smart meter } \\
\text { data access. }\end{array}$ & $\begin{array}{l}\text { Ireland (Beckel et al. } \\
\text { 2014) }\end{array}$ \\
\hline $\begin{array}{l}\text { Digital water meter at resolution of } 0.014 \text { litre per } \\
\text { minute (L/min) and data loggers ( } 5 \text { second (s) } \\
\text { intervals) }\end{array}$ & $\begin{array}{l}\text { Smart meter enabled informatics for economically } \\
\text { efficient diversified water supply infrastructure planning. } \\
\text { Using high resolution water end-use data to predict size } \\
\text { and scope of infrastructure upgrades. }\end{array}$ & Water & $\begin{array}{l}\text { Using modelling techniques and empirical input } \\
\text { data, model runs showed deferred and } \\
\text { eliminated augmentations, as well as reductions } \\
\text { in infrastructure sizing for the water savings } \\
\text { scenarios compared to the baseline scenario }\end{array}$ & $\begin{array}{l}\text { Gold Coast, Australia } \\
\text { (Gurung et al. 2015) }\end{array}$ \\
\hline
\end{tabular}




\subsection{Big data informatics}

Emerging technologies and the associated big data informatics, once fully understood and exploited, are the truly "smart" components of a digital water, electricity or gas grid, and these informatics can be used for a wealth of applications (Stewart et al. 2013; Zhou et al. 2016). Intelligent metering uptake however, remains relatively slow, due largely to the unexploited benefits from the back-end of the smart grid, including meters and sensors.

Informatics applying a range of mathematical, statistical and rule-based approaches can be used to reveal important information on demand from the available data provided at second, minute or hourly intervals (e.g. Nguyen et al. 2015, Makki et al. 2015). Such information is powerful for government, utility and customer planning and decision making (Zhou et al. 2016, Erevelles et al. 2016). In the energy sector, in-home devices (IHD) such as visual displays, smartphones, or web-based portals fed by raw metering data, have been used for some time now as a demand management tool (Darby, 2010). IHD have the potential to combine energy data with information such as billing data, saved $\mathrm{CO}_{2}$, and consumption benchmarking; the goal being to supply consumers with more valuable and enriched information for energy savings (Pitì et al. 2017).

There are few papers that comprehensively discuss the applications and benefits of collecting this data concurrently from water, gas and electricity utilities, storing it within the same database, and correlating it together to extract even further useful data on demand. In particular, such an integrated database allows customers to unpack the water-energy nexus as described in the next section.

\subsection{Water-energy nexus}

Water-energy links related to the use of water is emerging as a key pathway for integration of water and energy retail services provision (Conrad et al. 2017). The advanced status of water, electricity and gas metering and data, has contributed to the current dynamic nature of research regarding links between water and energy of consumers. A range of priorities have been identified in this area including the need for "integrated water-energy data storage" enabling data-warehousing to capture full performance metrics (Kenway et al. 2013a). Big data informatics can be used at city-wide (Lundie et al. 2004; Hall et al. 2011; Lane et al. 2015) or household scales (Beal et al. 2012; Escriva-Bou et al., 2015; Binks et al. 2016; Hussien et al. 2017).

Such information can be used by utilities and customers to explore a range of efficient technologies and strategies that can be used to reduce household water and energy consumption and can underpin the decision making process for sustainable management of existing and new developments (Beal et al. 2012; Vieira et al. 2014a ). 


\section{Vision of an integrated digital multi-utility service provider}

\subsection{Potential for digital multi-utility}

Utility retailers do not need to own the capital-intensive generation/supply (e.g. power plant for electricity) or distribution (e.g. pipe network for potable water) assets. Traditionally, a utility retailers' role has been to purchase utility resources from such asset owners and sell them to customers at an agreed price. However, as Google ${ }^{T M}$ and Amazon $^{T M}$ have shown, by having a direct relationship with numerous customers and accessibility to associated information, they can exploit new business opportunities.

Deregulation of the energy sector prompted the creation of many private electricity and gas retailers. The water sector is still largely government-owned with only a few international examples of privatised water retailers (e.g. Thames Water in the United Kingdom). Hence, utility retailers have had little opportunity to be innovative due to highly restrictive regulation, a risk adverse quasi-government work culture, and the lack of information available from existing manually read meters.

However, the advent of intelligent metering and monitoring technologies for utility services coupled with 'big data' analytics made famous by companies such as Google ${ }^{T M}$, creates significant opportunities for forward-thinking utility retailors (Stewart et al. 2010). Moreover, as argued in this article, a company that can integrate such technologies and concurrently collect resource demand data across the customers' utility services and provide user-friendly information platforms to feed this information back to customers and utility organisations, will reap the benefits of derived operational synergies and access to potentially extensive mass markets.

The primary benefit to a multi-utility digital retailer is access to intelligently processed and synthesised customer 'big data'. From such data, digital multi-utilities can for example create innovative tariff structures, manage peak demand, unpack the water-energy nexus, and derive innovative tailored resource conservation products and rebates. The scale of customers served, multi-utility services offered, and data-driven value-adding to the entire utility generation/supply/distribution grid system, means that the utility can optimise the management of the system being used, potentially providing extensive financial capital and operational benefits, and the customers can benefit from significantly lower overall utility bills.

Multi-utility service providers may even offer to install advanced metering technologies and information systems without the expectation that the small retail margin must be recovered from their initial capital investment. The long-term goal of these providers may be to build new global companies that use informatics to create new business opportunities such as informing customers that they have a significant water leak in their hot water system and providing details of qualified plumbers in their 
suburb, taking a commission from the plumber contracted. The visioned digital multi-utility retailer can thus exploit new business opportunities while providing financial and non-monetary benefits to their customers. But with digital utility transformation also comes a range of technological, societal and regulatory challenges that must be overcome, explored later in this section.

\subsection{Digital multi-utility system architecture}

Figure 1 shows a basic schematic architecture for a novel digital multi-utility meter, communications network and information system arrangement. It shows water, electricity and gas meter data will be transferred via a communications network (e.g. RF-mesh architecture with 5G base stations or via LoRaWAN, Sigfox, etc.) to a server containing a database with aligned interval data for water, electricity and gas demand. The information system also includes data processing modules that analyse individual and combined (i.e. water-energy) resource demand trends and creates user-friendly reports to stakeholders (i.e. utility, customer, etc.). An integrated multi-utility interface can be developed that is accessible by both the utility officers and customers, with user-oriented modules accessible to them for their particular functions and concerns.

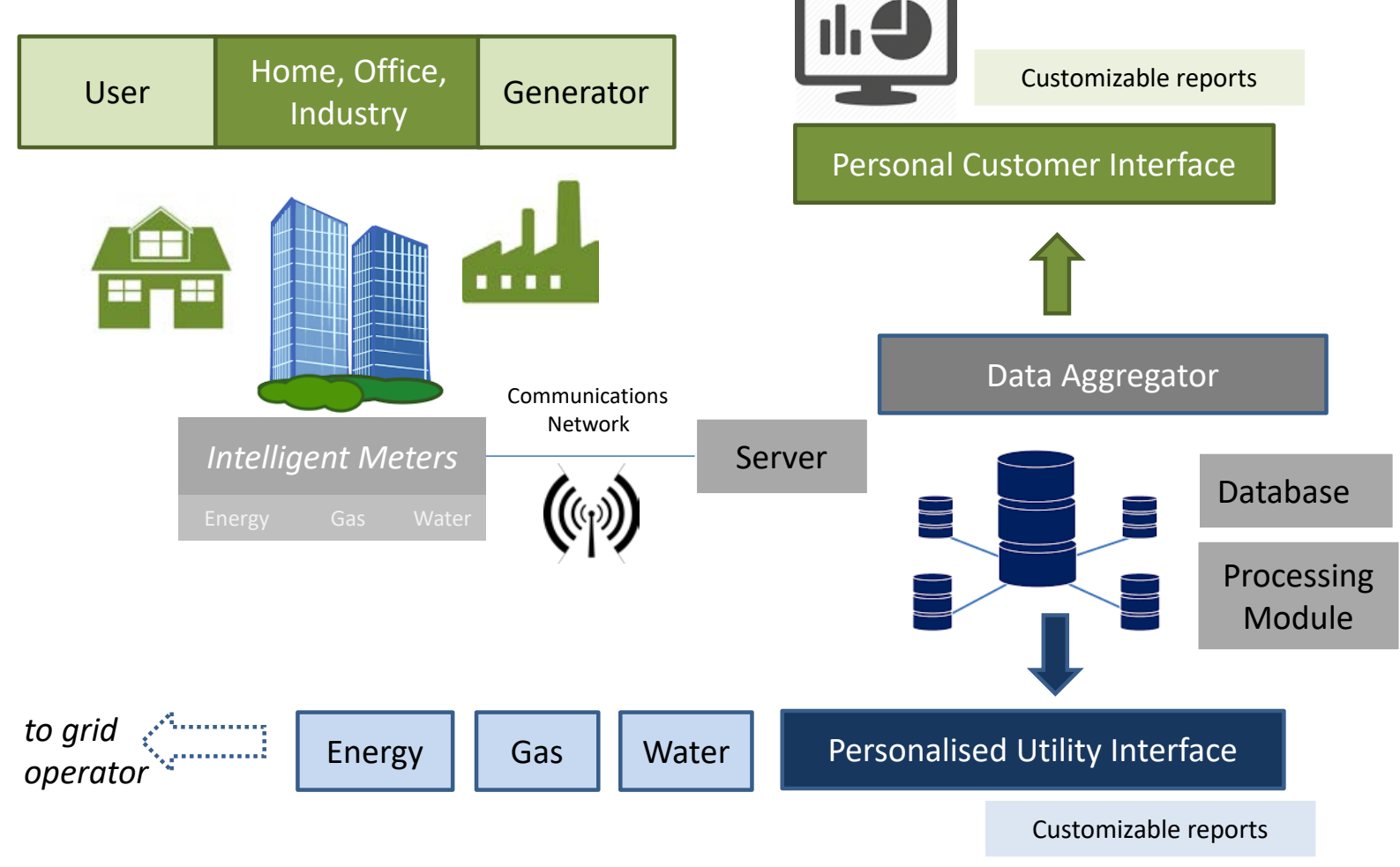

Figure 1. Digital multi-utility system architecture overview 


\subsection{Digital meter data opportunities and benefits}

Digital meters generate detailed interval data about water, electricity or gas resource consumption and time of use, yielding the overarching benefits of improved measurement, monitoring and management. However, to maximise the value of the data from such meters, it needs to also be connected to a database of relevant customer descriptive information. This combined database adds a distinctively new dimension to resource management by providing far greater insight into usage patterns, thereby offering new opportunities to create resource use efficiencies and savings for customers. Multi-utility data collection facilitates a long advocated an integrated resource planning (IRP) perspective (Turner et al. 2010).

Using a more holistic IRP approach, first developed in the electricity industry in the US and subsequently in the water industry predominantly in the US and Australia (Turner et al. 2010), assists in viewing how such advanced meters and data can be used. Whilst IRP has been used in water planning and management for many years (Turner et al. 2010) it has only been used to explore the opportunities of digital metering and analytics to a limited extent, that is, exploring the benefits from planning, demand forecasting and options analysis, through to implementation and monitoring and evaluation (Turner 2015; Turner and White 2017). This more holistic view is essential if we are to make the most out of such advanced meters, data and associated technology and behavioural interfaces within the water industry alone, without considering the vast opportunities of combined water, electricity and gas metering and analytics that add a whole new dimension to what is possible in utility resource management. The following sections discuss the opportunities and benefits of water, electricity, gas, and concurrently collected data.

\subsubsection{Water demand information}

Whilst not yet used as extensively as for electricity, water demand information from intelligent meters is being increasingly utilised and has significant opportunities for the water industry and its customers (Stewart et al. 2010; Boyle, et al. 2013; Cominola et al. 2015). For example, digital meters and associated informatics can help re-engineer out-dated demand modelling approaches by understanding customer usage patterns at far greater depths, such as an end use, and facilitate more efficient pipe network infrastructure planning (Gurung et. al. 2014; Gurung et al. 2016; Cominola et al. 2015; Creaco et al. 2016) as well as overall demand forecasting and strategic resource infrastructure planning.

In leakage and usage anomaly detection, traditional metering technologies often do not allow rapid and automatic detection of consumption changes due to the limited sampling resolution (e.g. litres consumed collected through monthly manual readings). Conversely, real-time detection systems based on intelligent metering offer great potential for water savings and money for both the utilities in the case of network leakages/anomalies on the distribution side (Puust et al. 2010), and for the end users in 
the case of leakages/anomalies on the customer side of the meter (Britton et al. 2013). Identifying and preventing anomalies could be provided by utilities as an extra, on-demand, service to customers, possibly coupled with complementary mitigation (e.g., prompt plumber intervention in the case of leaks) or compensation options (e.g., leakage insurance plans).

A number of studies have shown the benefits of using smart meter data for informing customers about their water demand and reinforcing efficiency efforts (Willis et al. 2010; Liu et al. 2015; Liu et al. 2016; Fielding et al. 2013; Sønderlund et al. 2016), now increasingly disseminated through smart phones and web-based applications not feasible only a few years ago. Going further, the combination of high resolution data with advanced disaggregation algorithms (e.g., Nguyen et al. 2013a) and estimated demand at the end-use level allows utilities and customers to consider the shift towards high-efficiency devices. With the cost compensated by the associated savings in terms of consumption on the customer side (Willis et al. 2013), sometimes water and energy, and deferred capital and operating expenditure for the utility, often amounting to millions of dollars (Turner et al. 2010). This shift to higher efficiency devices, possible through analysis and market segmentation, allows for targeted demand management programs (Liu et al. 2017) and subsequent almost instantaneous evaluation (Turner 2015).

\subsubsection{Electricity demand information}

In the electricity field, a number of works have pioneered the use of information from digital meters to support demand management (Siano, 2014) and increasingly diversified energy generation sources (Katsanevakis et al. 2017). Literature demonstrates that technological development of advanced metering systems such as smart plugs and distributed sensing networks (Morsali et al. 2012; Kobus et al. 2015), and smart appliances have increased the ability to collect energy use data at very high resolutions, with pilots reporting on data collected with sub-daily sampling rate frequencies of $\mathrm{MHz}$ (Armel et al. 2013). Moreover, recent evidence indicates that customer feedback based on real-time information, such as the design and implementation of heterogeneous demand management strategies (Gaiser and Stroeve, 2014), financial incentives encouraging consumers' to switch to energy efficient appliances (Geller et al. 2006), and awareness campaigns informing residential consumers about their energy use over time (Vassileva and Campillo, 2014), all induce energy savings and behavioural changes in the residential sector (Fisher, 2008).

Building on these promising outcomes, the use of electricity data from intelligent meters has been used to research Non-intrusive Load Monitoring (NILM) algorithms (Bennett et al. 2014; Bennett et al. 2015). Overall, development of intelligent meters and NILM algorithms can generate benefits for all parties involved. A study by Armel et al. (2013) reports that around 4\% of annual energy savings can be achieved simply by enhanced billing information, potentially rising to over $12 \%$ with informed realtime, appliance-level, feedback. Information from intelligent meters can allow energy users to get remote (historical) and immediate (real time) feedback about their use and associated information (e.g. 
cost, carbon emissions), along with technological and behavioural recommendations to increase efficiency (Faruqui et al. 2010), transparent information on their energy bill, and energy and costs savings (Ehrhardt-Martinez et al. 2010), similar to water sector application. Again, as with the water sector, utilities, in turn can acquire first-class data to support ongoing management. For example, for the design of diversified demand management and marketing programs, as well as tailored services to their customers, through informed customer segmentation (Albert and Rajagopal, 2013), increased operational efficiency (e.g., through peak demand reduction) and reduced costs and unnecessary electricity generation (Faruqui et al. 2010).

\subsubsection{Gas demand information}

The major domestic uses of gas include space heating, cooking and hot water. The gas consumption monitored using smart-meters in European countries (Joachain and Klopfert, 2014; Castelnuovo and Fumagalli, 2013) has provided detailed feedback to customers and utilities, which have been used for the accurate prediction of gas demand, optimal operation of utilities and efficient planning of government infrastructure. Recently, the smart grids for water, natural gas and electricity using advanced metering technology have been installed for efficiently managing the energy consumption of households, which enables the sustainable planning of infrastructure.

However, the sampling resolutions for gas metering are still very low. For instance, Squartini et al. (2015) employed sampling intervals of 1, 6, 12 and 24 hours to predict the natural gas demand. Olivera et al. (2016) detected gas leakage using a sampling interval of 1 minute. Little was reported on the highresolution (with sampling interval in seconds) digital metering and its impact on gas demand prediction. High-resolution sampling in gas metering is critical as gas data can be correlated with other similar interval data from water and electricity grids to obtain a more comprehensive picture of water and energy demand in a household.

For example, for instant gas boosted hot water, the correlation of gas use with water use for discrete events allows for a direct water-energy nexus calculation for showering and some washing machine and tap events. Furthermore, climate data such as the temperature and humidity may be integrated into the intelligent algorithm to improve the gas demand forecasting, as the consumption of space heating depends on air temperature and humidity (Fagiani et al. 2016) and the gas consumption of instant hot water is also temperature related.

Gas metering collected concurrently with electricity demand information can also allow a determination on the extent to which gas can reduce peak electricity demand; this is potentially a key strategic benefit of having a gas supply for certain energy requirements in a home. However, all these advanced analytics require high-resolution gas metering. 


\subsubsection{Concurrent resource demand information}

The examples above show that the water, electricity and gas sectors are exploring similar capabilities and objectives with their sector specific intelligent meters. Researchers have been considering the intricate water-energy nexus at a household level (Beal et al. 2012; Binks et al. 2016; Hussein et al. 2017) for several years, but due to a lack of concurrently collected high-resolution multi-utility data, they have had to rely on disconnected datasets or traditional empirical models to connect water and energy demand. The key benefits of having data from multiple utility sources are that the information can provide a paradigm shift in opportunities for load management and efficiency, customer profiling across time of use and for generating new business opportunities based on personalised demand and use profiles, for both residential and non-residential customers. It also has the potential to inform a future complex utility landscape where customers may be both generators and users of energy and even water - the so-called 'prosumers'. With new distributed digital database technologies like 'Blockchain' (Gadekar and Chandgude 2017) (www.blockchain.com), a new era in peer-to-peer trading and exchange has begun. Illustrative examples and case studies demonstrating the benefits of concurrently received medium and high resolution water, energy and gas consumption data, is detailed in later sections of this paper.

Recent worldwide applications (Cominola et al. 2015; Zeifman and Roth, 2011) have shown that the advent of digital metering technologies coupled with state-of-the-art informatics and data analytics can play a major role in supporting smart demand-side management solutions, 'ubiquitous optimization' and other related business improvements and opportunities.

The use of combined utility advanced data-mining algorithms that support the identification of recurrent routines in consumption data, and cluster users with similar habits (Kwac et al. 2014), could facilitate detailed segmentation and the design of customized demand strategies targeted to the specific features of each group of users, such as block tariff strategies tailored to the peak hours of a specific group of users. Moreover, customer segmentation and information on user habits can support utilities' marketing strategies aimed at offering tailored products and services, as well as improving individual customer experience (similarly to what online resellers such as Amazon are doing by advertising goods to users based on their past choices and habits).

Similarly, information from end-use disaggregation can support marketing and tailored strategies, not only for utilities, but also for appliance producers. Thus, ownership of high resolution, disaggregated, big data is a key asset for utilities fostering commercial partnerships with providers of complementary products/services requiring characterization of customer segments. This then leads to the need for the construction and maintenance of cloud systems able to safely transmit, store, and manage big amounts of data, opening up business opportunities for Telco's, cloud providers, and cyber security companies. 
Common tariff structures differ between utilities, for example flat or inclining block-tariffs in water and gas (but not dependent on time of use) and flat or inclining/declining block for electricity, perhaps with a peak/off-peak rate. Smart electricity meters also enable the development of more sophisticated time of use prices (see for example Surles and Henze, 2012, Wang et al. 2014). Depending on the utility and jurisdiction, the bill cost can be dominated by the fixed connection charge, the usage charge or a split between both. The installation of local energy (e.g. rooftop solar) can lead to feed-in tariffs where electricity generated by the consumer can be sold back to the grid and in the case of local electricity trading, directly to other consumers (Poullikkas, et al. 2013). Consumers who generate their own electricity (and then purchase less from the grid) can contribute to the 'death spiral' (Graffy and Kihm, 2014) where the costs of the fixed connection must rise to compensate the utility for selling less electricity to a consumer producing their own energy, the price rise can then prompt the consumer to see the installation of battery storage as increasingly economic through to a point where they disconnect from the grid, leaving a smaller number of householders to shoulder the burden of fixed network costs.

The advent of a digital multi-utility will introduce an interesting dynamic into an already changing space and could offer the opportunity to optimise demand across each utility resource, for example the timing of using pumped water storage in the grid (or even at the household scale), the potential for households with solar and batteries to act as on-demand virtual power plants and supply to the grid to meet peak demand and possibly even hybrid heating systems which can run from gas or electricity. An important consideration in developing new tariff structures is the impact on lower income households (Estache et al. 2001). A future scenario where large global digital multi-utility retailers are the norm and have significant market domination, they must be carefully regulated and managed to ensure that they are fairly serving lower income customers.

\subsection{Digital multi-utility impediments and challenges}

Notwithstanding the benefits of transitioning to a digital multi-utility, there are a number of impediments and challenges that need to be overcome before this vision can be realised. A comprehensive review of the literature identified the following categories of challenges with associated coping strategies to address them and achieve the goal of a digital multi-utility: (1) metering technology; (2) communications; (3) network; (4) cyber security and privacy; (5) societal; (6) economic and financial; (7) regulatory; and (8) other. A summary is provided in Table 2. A detailed discussion of each of these challenges and coping strategies is outside the scope of this position paper. 
Table 2. Summary of challenges and coping strategies for the digital multi-utility

\begin{tabular}{|c|c|c|c|}
\hline Component & Challenges & Coping strategies & References \\
\hline $\begin{array}{l}\text { Metering } \\
\text { technology }\end{array}$ & $\begin{array}{l}\text { - } \text { Meter battery life } \\
\text { - Poor signal, lagged data transfer, slow } \\
\text { response } \\
\text { - Tampering prone meters } \\
\text { - Signal transfer errors (e.g. reed switch } \\
\text { failure) } \\
\text { - Internal memory capacity } \\
\text { - Hall-effect issues }\end{array}$ & $\begin{array}{l}\text { - Tamper-proof mechanism } \\
\text { - Collaborations } \\
\text { vendors/utilities/research to } \\
\text { develop higher quality products }\end{array}$ & $\begin{array}{l}\text { Lloret et al. (2016); Keelson et } \\
\text { al. (2014); VICGOV (2014); } \\
\text { Ripka (2010) }\end{array}$ \\
\hline Communications & $\begin{array}{l}\text { - Lack of standards } \\
\text { - Interference among wireless systems } \\
\text { - Unaffordable proprietary wireless spectra } \\
\text { - Wired systems are expensive and hard to } \\
\text { - perform maintenance if required } \\
\text { - Short battery life } \\
\text { - Lack of interoperability among different } \\
\text { wireless protocols }\end{array}$ & $\begin{array}{l}\text { - Public cloud infrastructure } \\
\text { - LPWA emerging as affordable } \\
\text { connectivity option } \\
\text { - Selection of consolidated } \\
\text { protocols (e.g. Bluetooth) } \\
\text { compatible with previous } \\
\text { system but able to integrate new } \\
\text { media }\end{array}$ & $\begin{array}{l}\text { Gungor et al. (2011); Lloret et } \\
\text { al. (2016); Parikh et al. } \\
\text { (2010); Raza et al. (2017); } \\
\text { Soldatos et al. (2012); Wilson } \\
\text { et al. (2015) }\end{array}$ \\
\hline Network & $\begin{array}{l}\text { - Unfavourable site conditions for wireless } \\
\text { causing signal blockage or interference } \\
\text { - Cellular: not resilient during emergencies } \\
\text { - PLC noisy and low frequency data } \\
\text { transmission and hard to apply to services } \\
\text { other than electricity supply } \\
\text { - Density of signal repeaters }\end{array}$ & $\begin{array}{l}\text { - Flexible PLC supporting } \\
\text { different data rates } \\
\text { - } 5 \mathrm{G} \text { to provide low energy, } \\
\text { latency, bandwidth } \\
\text { - Hybrid solution required with } \\
\text { the selection of interoperable } \\
\text { systems tailored for different } \\
\text { conditions } \\
\text { - Mesh networks can be used to } \\
\text { increase routes for data } \\
\text { transmission }\end{array}$ & $\begin{array}{l}\text { Bahmanyar et al. (2016); } \\
\text { Cleveland (2008); Depuru et } \\
\text { al. (2011); Gungor et al. } \\
\text { (2011); Yan et al. (2013) }\end{array}$ \\
\hline $\begin{array}{l}\text { Cyber security } \\
\text { and privacy }\end{array}$ & $\begin{array}{l}\text { - Authentication, availability, } \\
\text { nonrepudiation, confidentiality, integrity } \\
\text { - Hardware and firmware manipulation } \\
\text { - Physical theft of meters and access to data } \\
\text { - Shielding antennas } \\
\text { - Easy to attack wireless sensors } \\
\text { - More encryption will add cost } \\
\text { - Hard to secure meters in anti-theft } \\
\text { locations } \\
\text { - Gateways managed by different entities, } \\
\text { difficult to agree on security technology }\end{array}$ & $\begin{array}{l}\text { - Develop proper security } \\
\text { standards } \\
\text { - Protocols with unique device } \\
\text { identification for non- } \\
\text { repudiation } \\
\text { - Coordinated security policies }\end{array}$ & $\begin{array}{l}\text { Cleveland (2008); Gungor et } \\
\text { al. (2011); McDaniel and } \\
\text { McLaughlin (2009); Rottondi } \\
\text { et al. (2013); Skopik et al. } \\
\text { (2012); Taormina et al. 2017; } \\
\text { Yan et al. (2013) }\end{array}$ \\
\hline Societal & $\begin{array}{l}\text { - Customers might not want to share data } \\
\text { - Smart meters perceived as complex } \\
\text { - Historically water, energy and gas } \\
\text { managed separately } \\
\text { - Wireless signal from smart meters } \\
\text { perceived as causing public health issues } \\
\text { - Privacy breach of consumption patterns } \\
\text { may be associated with legal and personal } \\
\text { security issues }\end{array}$ & $\begin{array}{l}\text { - Training personnel } \\
\text { - Programs to convince } \\
\text { customers of the benefits } \\
\text { - Collaborations vendors/utilities } \\
\text { to develop marketable products } \\
\text { - Stakeholders engagement } \\
\text { - Research integration } \\
\text { - Signal strength of wireless } \\
\text { systems within guideline } \\
\text { requirements }\end{array}$ & $\begin{array}{l}\text { Cheong et al. (2016); Khan et } \\
\text { al. (2017); Kim et al. (2014); } \\
\text { Kim et al. (2007); Lloret et al. } \\
\text { (2016); Paetz et al. (2012); } \\
\text { Parkhill et al. (2013); } \\
\text { Rohracher (2003) }\end{array}$ \\
\hline $\begin{array}{l}\text { Economic and } \\
\text { financial }\end{array}$ & $\begin{array}{l}\text { - Developers install the cheapest systems } \\
\text { for compliance with legislation and } \\
\text { handover to utility } \\
\text { - Current installation/maintenance costs too } \\
\text { high for utility } \\
\text { - No direct return of investment } \\
\text { - Current technologies either unaffordable } \\
\text { or limited capabilities } \\
\text { - Deployment of obsolete systems may } \\
\text { frequent upgrade requirement with high } \\
\text { cost implications } \\
\text { - Low data resolution of standard meters } \\
\text { not feasible for time-of-use tariffs }\end{array}$ & $\begin{array}{l}\text { - Incentives/subsidies } \\
\text { - Education to achieve cost- } \\
\text { sharing } \\
\text { - Internal training to avoid } \\
\text { outsourcing } \\
\text { - Specifications of meters must } \\
\text { be detailed enough to prevent } \\
\text { the installation faulty prone } \\
\text { system, and flexible enough to } \\
\text { enable the adoption of new } \\
\text { cutting-edge technologies with } \\
\text { improved financial feasibility } \\
\text { - Clear understanding of portfolio } \\
\text { of available technologies and } \\
\text { future trends for smart } \\
\text { metering. }\end{array}$ & $\begin{array}{l}\text { Bahmanyar et al. (2016); } \\
\text { Cheong et al. (2016); Dedrick } \\
\text { and Zheng (2011); Depuru et } \\
\text { al. (2011); El-hawary (2014); } \\
\text { Kaufmann et al. (2013); } \\
\text { Luthra et al. (2014); SGCC } \\
\text { (2013); Pitì et al. (2017); } \\
\text { Pallonetto et al. (2016); } \\
\text { Rogers and Carroll (2016). }\end{array}$ \\
\hline
\end{tabular}




\begin{tabular}{|c|c|c|c|}
\hline & & $\begin{array}{l}\text { - Tariff reform to include time- } \\
\text { of-use or real-time tariffs in } \\
\text { order to divert peak } \\
\text { consumption to off-peak hours } \\
\text { - Tariff reform to encompass } \\
\text { strategies to reduced } \\
\text { concomitant energy and water } \\
\text { peaks and optimise the } \\
\text { performance of utilities and } \\
\text { buildings. }\end{array}$ & \\
\hline Regulation & $\begin{array}{l}\text { - Policies are complex and open to contest } \\
\text { and negotiation } \\
\text { - Current policies are obsolete and } \\
\text { discouraging a change } \\
\text { - Policies incentivising consumption do not } \\
\text { motivate utility to change } \\
\text { - Political will required despite potential } \\
\text { economic benefits } \\
\text { - Lack of clear directions and } \\
\text { responsibilities may lead to ineffective } \\
\text { outcomes }\end{array}$ & $\begin{array}{l}\text { - Stakeholders engagement and } \\
\text { education } \\
\text { - Targeted research funding } \\
\text { - Financial incentives } \\
\text { - Robust solution to account for } \\
\text { uncertainty } \\
\text { - Demonstration projects }\end{array}$ & $\begin{array}{l}\text { Bahmanyar et al. (2016); } \\
\text { Bulkeley et al. (2016); Cheong } \\
\text { et al. (2016); Depuru et al. } \\
\text { (2011); El-hawary (2014); } \\
\text { Kaufmann et al. (2013); Khan } \\
\text { et al. (2017); Luthra et al. } \\
\text { (2014); Mutchek and Williams } \\
\text { (2014); Vojdani (2008) }\end{array}$ \\
\hline Other & $\begin{array}{l}\text { - High data frequency required for reliable } \\
\text { modelling }\end{array}$ & $\begin{array}{l}\text { - Address technical challenges to } \\
\text { overcome data frequency issue } \\
\text { - Integrate GIS with smart meters } \\
\text { for better faults location }\end{array}$ & $\begin{array}{l}\text { Bahmanyar et al. (2016); } \\
\text { Depuru et al. (2011); Khan et } \\
\text { al. (2017); Wilson et al. } \\
\text { (2015) }\end{array}$ \\
\hline
\end{tabular}

\section{Modelling contemporaneous multi-utility demand data from intelligent meters}

The coupled analysis and modelling of water and energy data has shown valuable state-of-the-art applications to inform single and multi-utilities and regulatory agencies. Indeed, low and medium resolution data can be exploited to perform urban scale studies aimed at assessing the environmental impacts and costs of water-related energy (Escriva-Bou et al. 2015), as well as exploring heterogeneous consumption patterns. In contrast, detailed end-use water consumption data requires higher resolution digital water meters, capable of measuring very low flow rates (e.g. $0.01 \mathrm{~L}$ ) at close logging intervals (e.g. 5s) (Giurco et al. 2008). In the energy industry where smart electricity and gas meters and communications infrastructure have already been more widely introduced, low-medium power consumption data collected at minute or hourly interval have been effectively used in power demand forecasting, or design of customized energy demand management strategies. Several algorithms for high-resolution power consumption end-use disaggregation have been proposed and summarised in a review paper by Zoha et al. (2012).

This paper advocates a vision of a multi-utility where demand data is concurrently collected and modelled to allow for enhanced pattern recognition of other resources (e.g. having electricity data assists pattern recognition of water), deeper insight into customer demand and strategies to manage it, as well better water and electricity grid infrastructure asset management. The following sub-sections discuss and illustrate how the collection and modelling of concurrent multi-utility data of different resolutions can be used by the multi-utility provider to benefit customers, utilities and regulatory agencies. 


\subsection{Concurrent modelling of medium resolution meter data}

Low resolution (i.e. monthly, quarterly) water and energy consumption data is still largely being gathered in modern economies, and this data has mainly been used for billing purposes. Considering modelling purposes, the use of such data has been limited to feed statistical time-series analysis and simple econometric models to estimate aggregate demand levels at the municipal or district level, or for assessing the macro-effect of exogenous variables (e.g., seasonality, water/energy prices) on demand at regional scale (House-Peters and Chang, 2011). Yet, low-resolution data provides restricted capability of representing the spatial and temporal heterogeneity of water and energy demands (Cominola et al. 2015). Moreover, the coarse data sampling frequency does not allow the implementation of quasi-real time management actions, as well as the detection of anomalous events and consumption behaviours (Loureiro et al. 2014a).

Finally, the exploitation of coupled water-energy data at low resolutions has been limited to accounting for energy costs associated with water pumping in water tariffs design (e.g., Spang et al. 2015), assessing the energy intensity of water treated and delivered to customers in large-scale urban areas (Spang and Loge, 2015), or developing models that rely on water-energy data and end-use parameters to assess water-related energy use, greenhouse gas emissions, and costs (e.g., Fidar et al. 2010; Kenway et al. 2015; Escriva-Bou et al. 2015). Medium-resolution data opens further opportunities for the development of fine-scale demand models. For instance, in the electricity sector Kwac et al. (2014) developed a customer segmentation technique able to discriminate among heterogeneous clusters of electricity users based on a set of typical 24-hour consumption patterns (i.e., load shapes) iteratively extracted from a database of hourly consumption data.

Similarly in the water sector, apart from allowing earlier detection of leaks and anomalies (Britton et al. 2013), recent works by Cardell-Oliver et al. (2016) and Cominola et al. (2017a) showed the potential of data-mining hourly water consumption data to identify recurring behaviours (routines) in the residential sector, and pinpoint links with demographic and household characteristics. The above examples represent promising approaches to uncover demand patterns and demand features useful to inform supply-side operations such as peak demand (Beal et al. 2016a), as well as heterogeneous categories of water or electricity users to advance the development of customized demand management strategies. However, there are sparse reported cases of data mining and analysis for coupled waterenergy datasets. One very recent study by Cominola et al. (2017b) performed data mining and customer segmentation on a coupled water-energy dataset, in order to explore opportunities for joint planning and management actions, and inform multi-utility operations and water-energy conservation programs. 


\subsubsection{Illustrative examples}

\section{Example 1: Assessing the energy intensity of water treated and delivered to urban customers}

A first example showing how low-medium-resolution water and energy data can be jointly exploited is provided by recent work by Spang and Loge (2015). In this study, the authors consider a mix of monthly and hourly resolution water and energy data, and develop a method for evaluating the energy intensity of water treatment and delivery processes for the service area of the East Bay Municipal Utility District in Northern California. The model presented takes into account seasonal and topographic effects impacting on water delivery (and related energy costs). Findings on how the energy intensity of water changes among pressure zones, seasons, and topography provide utilities and water agencies useful insights for infrastructure planning and design of water saving programs leading to related energy savings, at the urban scale. Yet, this large spatial scale study does not include information on heterogeneous consumers' behaviours or residential end-uses.

\section{Example 2: Assessing water-related energy footprint with end-use information}

A number of recent applications show that low-medium resolution water and energy data can be utilized to perform detailed impact assessment of residential water consumption, in terms of related energy (mainly for water heating) and consequent GHG emissions. These studies (Fidar et al. 2010; Kenway et al. 2013a; Escriva-Bou et al. 2015), coupled total household water and energy consumption data with daily or sub-daily characteristics of water end-uses. For example they used average flow-rate, volume of water per event, etc. in order to both assess the aggregate household impact, as well as the impact of each component on the total households' consumption, thus informing conservation programs on effective ways to reduce the overall direct and indirect impacts of residential water use.

\section{Example 3: Water-energy consumer segmentation for customized demand management}

The recent work by Cominola et al. (2017b) provides an example on how water and energy data can be jointly used to inform demand management actions by agencies and multi-utilities. The study contributes a customer segmentation analysis of hourly water and electricity data for over 1000 residential accounts in South California. This data analysis example is featured as a case study in a later section of the paper.

\subsubsection{Applications for utilities and regulatory agencies}

Based on recent studies showcasing modelling applications on low-medium resolution water-energy data, the following applications for utilities and regulatory agencies emerges: (1) low resolution (for instance seasonal or monthly) data can be used to develop seasonal pricing schemes or block pricing schemes based on total demand magnitude, in order to control water and energy demands during highuse periods, as well as reduce costs; (2) low resolution data can be used for detecting dominant changes 
in demand trends overtime, due to population increase, for instance; (3) low and medium resolution data can support the assessment of water demand impact on other sectors (e.g., energy production and supply, and environment), thus supporting strategic decision making for efficiency programs; (4) medium resolution data (i.e. hourly), supports comparative studies of water and energy demand patterns for average and peak days, and allows for the identification of demand peaks, correlations between water and energy demand patterns, and customer segmentation; (5) cross-correlation between waterenergy consumption data and consumers' and households' features allows identifying groups of target users for demand management interventions, as well as the drivers of their demand, thus supporting the design of customized feedbacks; and (6) hourly demand data can improve leakage and anomaly detection systems.

\subsubsection{Applications for customers}

On the customer side, the applications of medium-low resolution data are predominately related to more detailed communication of consumption feedback to consumers, and include: (1) users can monitor their daily or hourly water consumption data in quasi real-time and through easily accessible digital portals (e.g. Opower, iWIDGET and SmartH2O platforms); (2) users can be promptly informed and warned about anomalous consumption events and leaks; (3) customized feedback can be provided based on time-of-use or exceedance of high demand thresholds; (4) consumption levels can be visualized in contrast with data from peers, in order to foster efficient behaviours through peer-pressure mechanisms; and (5) users can be informed about the impacts of their water and energy demand and choices using various ecological footprint indicators (e.g. GHG).

\subsection{Concurrent modelling of high resolution meter data}

Concurrently collected high resolution water-energy data contains valuable information which, if thoroughly explored, provides a range of benefits for both utilities and customers. Disaggregated water and energy demand data into different end-use categories is one of the most notable of these benefits. Certain appliances in a household require both water and energy to function, such as a clothes washer and dishwasher, and understanding the water and energy demand of each individual event is of great value. It should also be noted that the disaggregation of demand data into end-use categories becomes more accurate when there are multiple signals relating to that appliance (i.e. water and energy signal). The below sub-sections illustrate how the water and energy demand of a single clothes washer water event can be pattern recognised using a single independent demand pattern (e.g. water pattern), and demonstrates how a concurrent water-energy pattern improves the accuracy of pattern recognition. Potential benefits for customer and water utilities from exploiting the concurrent high resolution data are also described. 


\subsubsection{Illustrative examples}

\section{Example 1: Clothes washer classification using individual water flow pattern}

A clothes washer is a very common appliance present in most households, and the classification of enduses like this is a focus of all studies relating to water and energy consumption disaggregation (Nguyen et al. 2015; Anderson, 2014). In terms of energy, smart meter data collected at minute or hourly intervals was used as the main resource, and several techniques have been applied to achieve this task, including genetic algorithms, integer optimization, sparse optimization, factorial hidden Markov model, dynamic time warping, signature-based algorithms, or hybrid algorithms (Zoha et al. 2012; Piga et al. 2016; Cominola et al. 2017b). In the water domain, data collected at several different resolutions was also used, and the maximum recorded accuracy of $90-94 \%$ for clothes washer classification was reported in (Nguyen et al. 2014) when several techniques including hidden Markov model, dynamic time warping algorithm, probabilistic models and artificial neural networks were all combined to analyse smart water data collected at 5 seconds intervals with a data resolution of 72 pulse per litre ( $0.014 \mathrm{~L} /$ pulse). The authors are not aware of other substantial research works seeking to use multiple concurrently collected utility demand signals to aid the pattern recognition of other signals.

\section{Example 2: Clothes washer water-energy use classification using concurrent data patterns}

A demonstration of how the classification of end-uses can benefit both joint water and energy data is described here through the classification of clothes washer use events using concurrent water-energy signals, and comparing the outcomes against those obtained from separate water or energy data. In a pilot project of a single household, 5 second data was concurrently collected for both water and energy use data (see Figure 2). The figure illustrates a strong correlation between the water (green) and energy (blue) signal for the overlaid patterns. More specifically, it indicates that during the spin cycle of the clothes washer, water is injected while power is used to spin the drum, which resulted in two closely aligned signals. Closely correlated water-energy related pattern features such as these are important as they significantly enhance the classification accuracy of utility demand events for both water and energy, which is particularly useful for times of peak water and energy demand. 


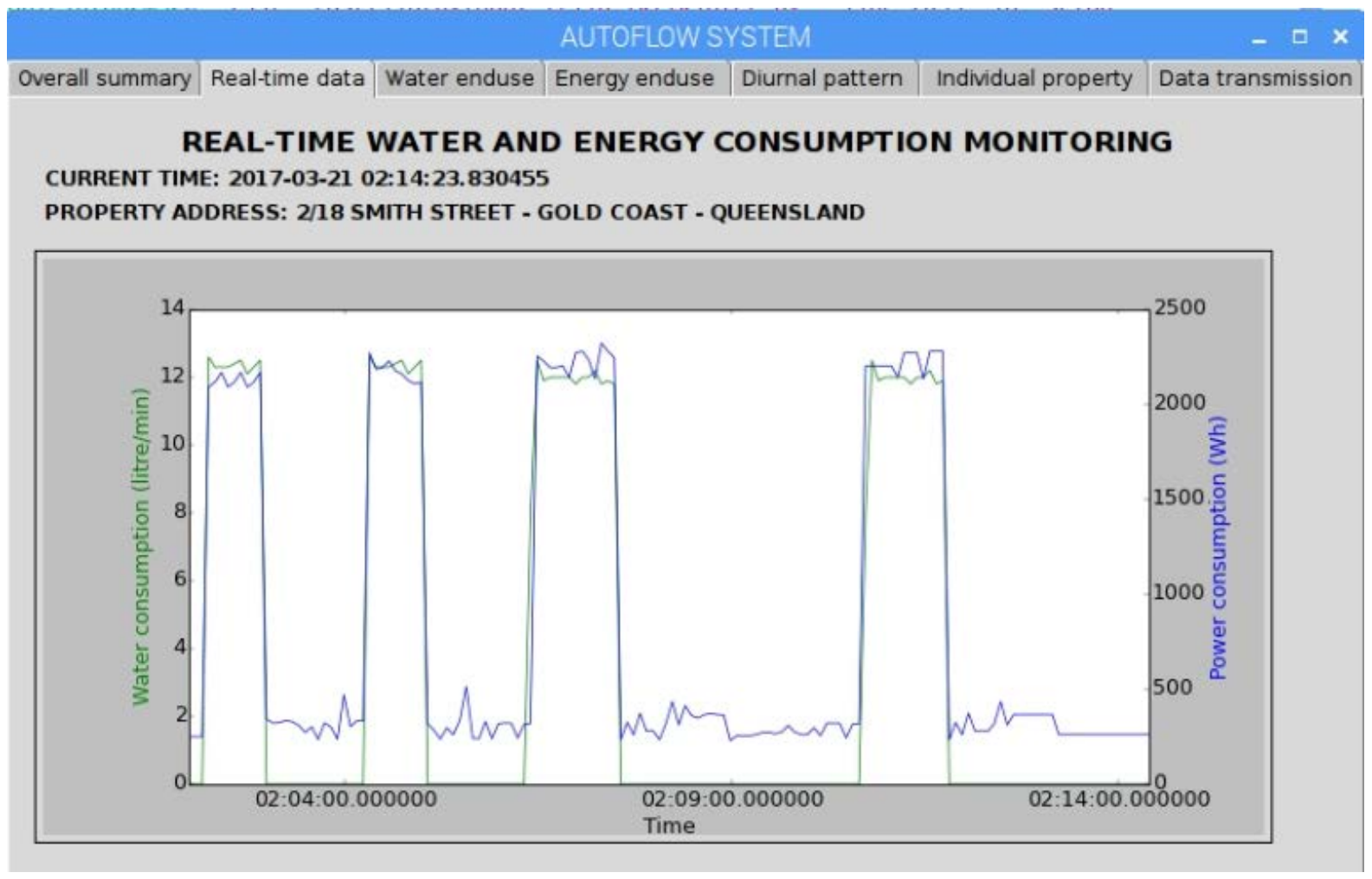

Figure 2. Concurrent water-electricity demand signal (5s; L/min and Wh) (Autoflow screen shot)

When only water data is provided, the clothes washer pattern sometimes has similarities with some toilet or tap event patterns. The addition of concurrent energy consumption patterns improves the accuracy and efficiency of both the water and energy pattern recognition process. Moreover, by improving the pattern recognition accuracy of events that consume both water and energy, the recognition accuracy of separate energy (e.g. oven) or water (e.g. toilet) events will be improved through greater deductive reasoning. Water-related heating (e.g. instant gas boosted showers) is another end use category where there is a strong correlation between water and energy patterns.

Figure 3 illustrates a screen shot of a prototype 'Autoflow' system that wirelessly collects real-time high resolution water and energy signals ( $5 \mathrm{~s}$ interval) and performs on-board data analysis and processing to disaggregate water and energy consumption into different water and energy end-use categories. An illustrative example is provided for a single property in Figure 3. The Autoflow software was originally developed for the purpose of autonomous water end-use classification Nguyen et al. (2011; 2013a; 2013b; 2014; 2015) but recent developments of this software and its underpinning algorithms have been focused on enhancing this software to unpack both water and energy end-use demand components. End use data has a range of applications for both customers (e.g. feedback on demand) and multi-utilities (e.g. understanding end uses contributing to peak demand). 


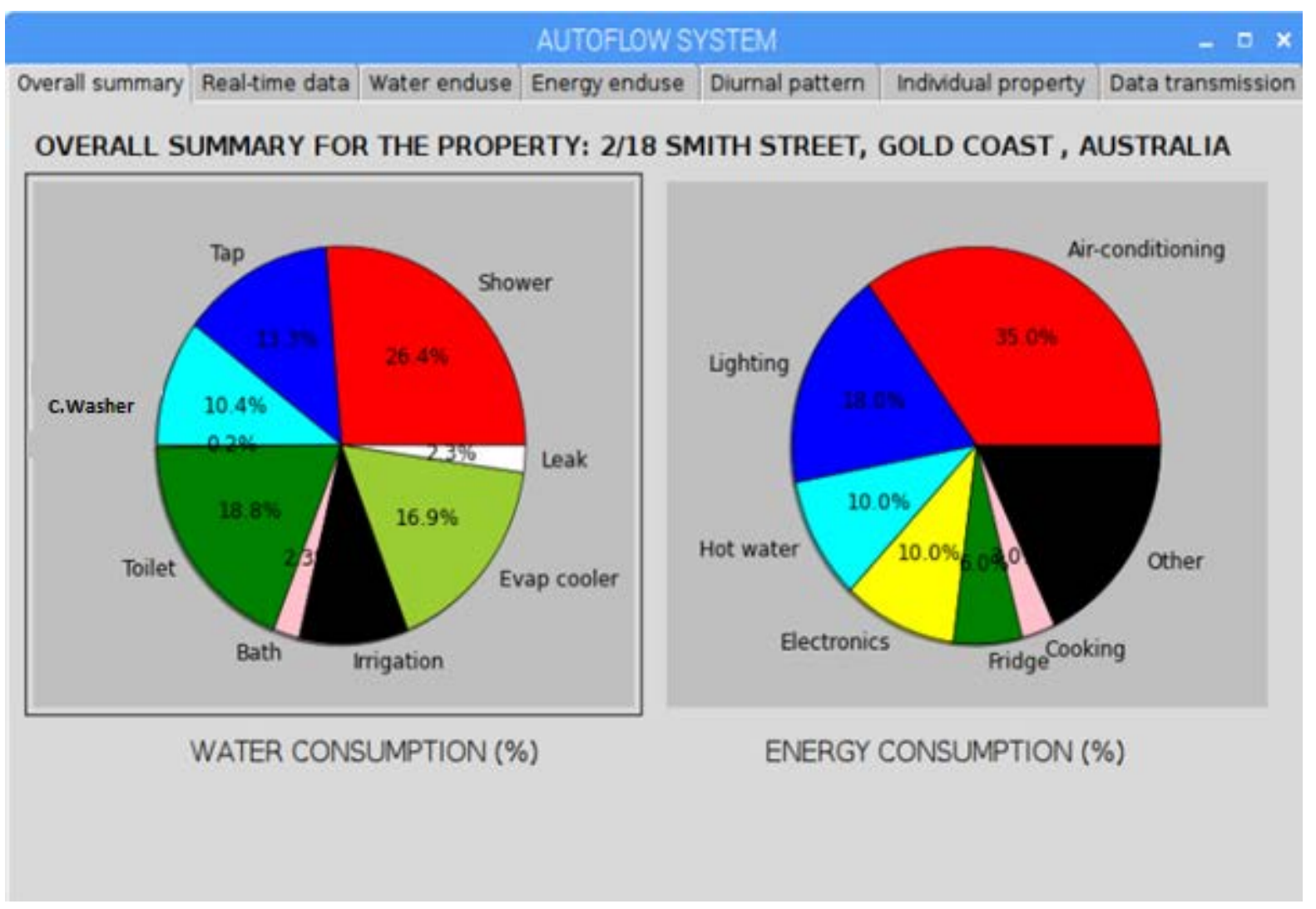

Figure 3. Water-energy demand end use summary for single home (Autoflow software screen shot)

\subsubsection{Applications for utilities and regulatory agencies}

The collection and analysis of concurrent water and energy data will result in a wide range of benefits for utilities and regulatory agencies. This includes: (1) having a unique single platform to monitor all water and energy consumption of any particular household or region in near real-time to immediately identify issues (e.g. household water leaks); (2) optimised water and energy grid infrastructure asset management; (3) improved customer satisfaction when user is provided with comprehensive information regarding the efficiency of their appliances (e.g. "your clothes washer water and energy consumption per load are $140 \mathrm{~L}$ and $1300 \mathrm{~W}$, which is much higher than the average consumption of $70 \mathrm{~L}$ and $800 \mathrm{~W}$ '); and (4) provides utilities and regulators to develop more effective demand management messaging and strategies (e.g. rebates, education, restrictions, etc.) during periods of water scarcity or peak energy periods by using information derived from the concurrent data.

\subsubsection{Application for customers}

Customers will gain significant benefits from the analysis and presentation of concurrent water-energy data from a multi-utility provider, including: (1) one single account to view both real-time water and energy consumption as well as other statistical reports, including comparisons with other households with similar demographic patterns, notification from suppliers, detailed end use disaggregation, or 
recommendations to help reduce consumption; (2) be immediately alerted when water or energy demand is uncharacteristic (e.g. water leak in home); and (3) be informed about the current efficiency status of water and power appliances and devices; (4) receipt of rewards when shifting or reducing demand on the request of the utility to achieve certain supply or distribution grid infrastructure management objectives (e.g. Message from utility: "Please avoid using clothes washer and dishwasher between 6 to 8pm tomorrow. In return, you will receive ten reward points to your account. Once 50 points has been accumulated, you will receive a \$10 discount on your next bill”).

\section{Case studies showcasing applications of concurrent water-energy data}

Together with socioeconomic and competitive market pressures, advances in digitalisation and technological innovations are the key drivers behind the development of digital multi-utilities. Globally, the adoption of smart technologies has been increasing rapidly resulting in the massive generation of data together with an increasing ability of utilities to collect, process, analyse and use multiple data points. These trends are bolstering the feasibility of the digital multi-utility combining multiple-services by connecting data and technology to increase efficiency and consequently, make the multi-utility concept attractive to both utility operators (e.g. network asset management) and customers (e.g. potential tariff reductions). Concurrent water/energy data collection and processing would create synergy in improving performance in utility administration and cost reduction through integrated tailormade services to their customers. Thus, the formation of digital multi-utility retailers can enable utilities to improve their productivity and efficiency by using high-resolution smart meter data for all measurements and analytics.

This section provides an overview of four case studies that demonstrate just a few of the many applications and benefits of concurrently collected water and energy data. These case study demonstrations seek to showcase the potential widespread implications of the digital multi-utility. Case study 1 demonstrates how the simultaneous collection of high resolution data of water and energy for individual end use events supplied by a rain tank enabled better understanding of the energy intensity of those end uses. Case study 2 demonstrates how the simultaneous collection of medium resolution data can be used for customer segmentation analysis of residential accounts of water-electricity consumers. Case study 3 summarises a household level analysis of water-energy nexus data to better understand shower end use consumption trends. Case study 4 showcases a European case study where a prototype digital multi-utility web interface containing key water-energy feedback information has been provided to customers to inform them of their consumption trends, conservation opportunities and demand anomalies. 


\subsection{Case study 1: End use level water-energy analysis of residential rain tanks in households}

\subsubsection{Case study overview}

To tackle potential water shortages, many countries have been working on developing alternative water management strategies such as decentralised (e.g.: rainwater harvesting) or rain-independent water sources (e.g. desalination plants). Decentralised rainwater harvesting systems were often considered as a good solution to provide a substitute to traditional centralised reservoir supplies (Siems and Sahin, 2016). For example, as at 2012, 35\% of new buildings in Germany were built with a rainwater harvesting system (Galbraith, 2012). In the UK, rainwater harvesting is encouraged by the Code for Sustainable Homes (Environment Agency, 2010). Similarly, many Australian State and Local governments mandated the installation of internally plumbed rainwater tank systems (IPRWTS) during the severe drought across Australia. As a result, 2.3 million households had an IPRWTS (Australian Bureau of Statistics, 2013), about $50 \%$ of which had an internally plumbed IPRWTS. Typically, IPRWTS in Australia included the installation of a $5 \mathrm{~m}^{3}$ rainwater tank, pressurised with a single speed pump and were plumbed internally to toilets, garden taps and clothes washer fixtures (Siems and Sahin, 2016; Stewart, 2011). Without gravity head, most of these systems require a pump to supply captured rainwater to end-uses. These pumps are mostly energy intensive during their start-up and throughout a water use event (Talebpour et al. 2014). Despite the efforts focusing on alternative water substitutions in urban areas, the energy intensity of these substitutions has typically not been examined adequately by considering both economic and environmental factors in order to maximise the effectiveness of these strategies in the long term (Retamal et al. 2008; Proenca et al. 2011; Vieira et. al. 2014b; Siems and Sahin 2016). Further, the previous studies are limited to finding the total amount of energy used by an IPRWT as they examined the energy intensity of the IPRTWS only at an overall system level rather than investigating at an end-use level (i.e. toilet, clothes washer, outdoor tap). Using high resolution smart water and energy meters, Talebpour et al. (2014) and Siems and Sahin (2016), were able to capture 5 s interval data of water $(0.014 \mathrm{~L} /$ pulse) and pump electricity usage $(0.1 \mathrm{Wh} /$ pulse $)$ that enabled the determination of the energy intensity of individual end-uses (i.e. toilet, clothes washer, irrigation) supplied by IPRWTS as detailed below. This case study demonstrates the capability of the multi-utility and its customers to better manage complex decentralised water supply systems.

\subsubsection{Approach and findings}

The main objectives of these two studies conducted by Talebpour et al. (2014) and Siems and Sahin (2016) were to (i) gain an in-depth understanding of the water and energy requirements of IPRWTS supplied end-uses by using data collected from households in Gold Coast City, Australia; (ii) analyse the energy intensity of IPRWTS at an end-use level (toilet, clothes washer and irrigation) and (iii) understand the energy intensity and the associated economic and environmental implications of the IPRWTS. 
19 households with IPRWTS (broadly representative of the cross section of households in the region) were randomly selected to continuously collect high-resolution water and pump energy data (readers are referred to Talebpour et al. 2014 and Siems and Sahin, 2016 for further detail). The water and energy usage data collected through smart meters was disaggregated into four end-use categories (clothes washer, irrigation, toilet half-flush, toilet full-flush) using Autoflow ${ }^{T M}$ software. In addition, an extensive demographic and water-energy appliance stock survey were conducted to reduce classification uncertainty. From a data population of 1,210 events captured during the six-month monitoring study, the following aspects could be evaluated:

Water and electricity demand relationships for the IPRWT supplied end-uses: As illustrated in Figure 4(a), high flow rate events are more efficient than low flow rate events. Both Event 1 and Event 2 have a median electricity supply rate of $0.16 \mathrm{Wh} / \mathrm{s}$, however, their rainwater supply rates are significantly different, $0.174 \mathrm{~L} / \mathrm{s}$ for Event 1 and $0.036 \mathrm{~L} / \mathrm{s}$ for Event 2, which is 5 times more efficient than Event 1. This means that high-flow rate events exhibit the lowest energy intensity due to the pump system working closer to its optimal pumping range, with efficiency only lowered at the beginning and end of an event (Siems and Sahin, 2016). Thus, the selection of high flow rate appliances and irrigation equipment could lead to a substantial reduction in electricity costs for homeowners (Talebpour et al. 2014). Alternatively, lower power output, pressure tanks and/or variable speed pumps should also be considered by home-owners instead of selecting the commonly used fixed speed pump (mostly 700$800 \mathrm{~W})$.

Energy consumption at an end-use level and a comparison of energy intensity of events: As exhibited in Figure 4(b), the half-flush toilet category has the highest average energy intensity (1.9 Wh/L) but the lowest average water and energy consumption ( $3.2 \mathrm{~L}$ and $5.8 \mathrm{Wh}$ ) while the irrigation category has the lowest energy intensity (1.1 Wh/L) and highest average water and energy consumption (249.9 L and 263.4 Wh). High energy intensity of half-flush toilet events is a result of frequent starts and stops of the pump for a very short period of time, a low flow rate and a relatively small volume of water. In contrast, irrigation events typically have a long duration, higher volume and higher flow rate, which provide an ideal condition for an optimal pump performance.

Stand-by (non-event) energy consumption: Non-event energy consumption (NEC) refers to energy usage not directly associated with rainwater supply. A varying amount of energy is used for pumping water for each individual end-use event. Besides, rigorous analyses of the raw data feed showed that some IPRWTS had been consuming electricity when no water was supplied. This NEC is mainly attributed to toilet cistern overflow leaks. Site inspections and data analysis revealed that $58 \%$ of the installed systems consumed less than $0.1 \mathrm{kWh}$ per month when not supplying rainwater (Group 1 ) while other $42 \%$ (Group 2) consumed a proportionally large amount of energy constituting $35 \%$ of the total pump electricity used over the 6 month period. Consequently, as shown in Figure 4(c), the total energy 
intensity for the Group $2\left(2.26 \mathrm{kWh} / \mathrm{m}^{3}\right)$ is much higher than the Group1 systems $\left(1.29 \mathrm{kWh} / \mathrm{m}^{3}\right)$ as the latter systems did not consume power when unused.

Life cycle water-energy-carbon assessments: To assess the importance of IPRWTS energy intensity, the electricity, water costs and $\mathrm{CO}_{2}$ emission were calculated over a 20 year simulation period under 3 performance scenarios, namely, the average, most efficient and least efficient IPRWTS (Siems and Sahin, 2016). The most efficient system refers to a system with high flow rates for all end-uses, an efficient pump and no NEC. The least efficient system represents a system with low flow rates for all end-uses, an inefficient pump and leaky toilet cisterns leading to NEC. Results from the simulations are illustrated in Figure 4(d). Over a 20 year period, the least efficient system required double the electricity costs of the most efficient system, based on current prices. Moreover, as shown in Figure 4(d), the least efficient system will generate about 1.25 tonnes more $\mathrm{CO}_{2}$ than the most efficient system over a 20year period.

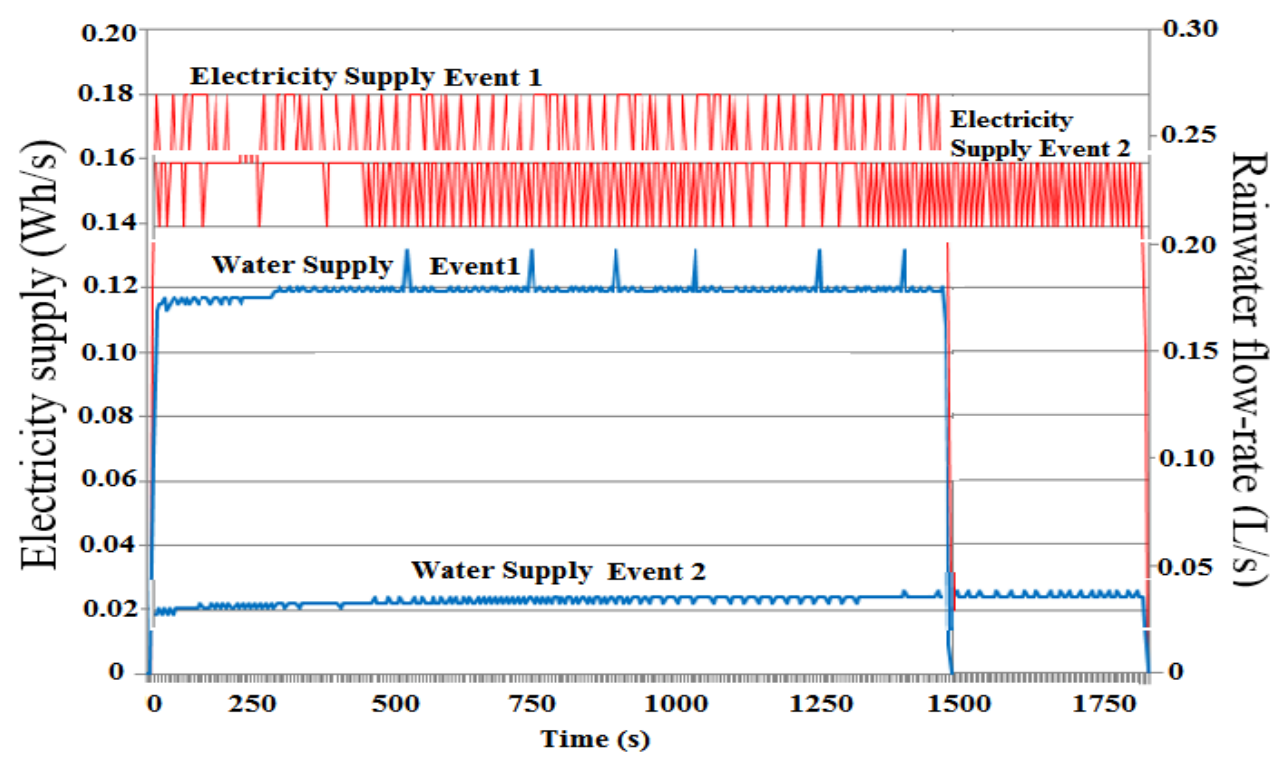

(a) Pump performance comparison from two irrigation events taken from the same system 


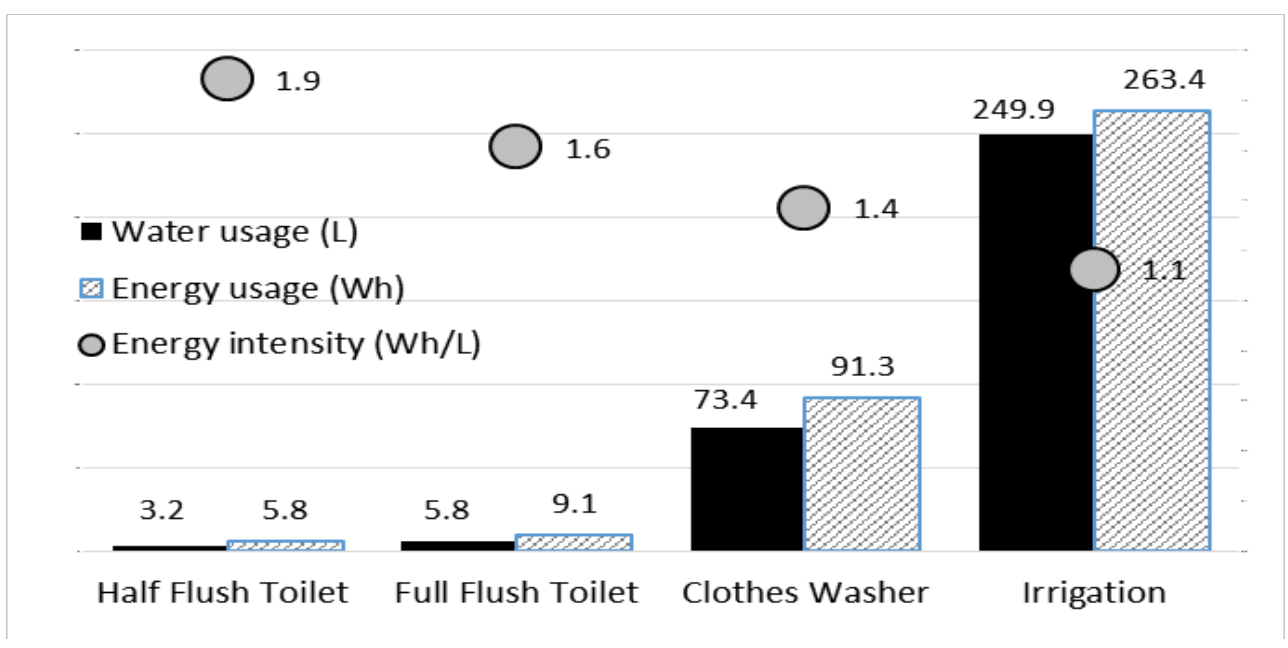

(b) Average energy intensity and energy and water usage at an end-use level

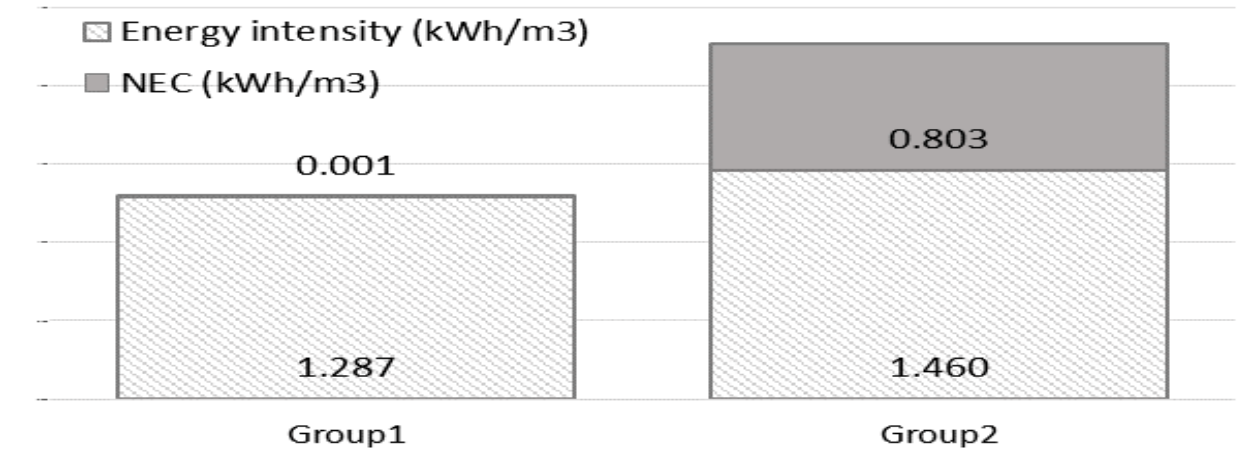

(c) Pump NEC and total energy intensity $\left(\mathrm{kWh} / \mathrm{m}^{3}\right)$

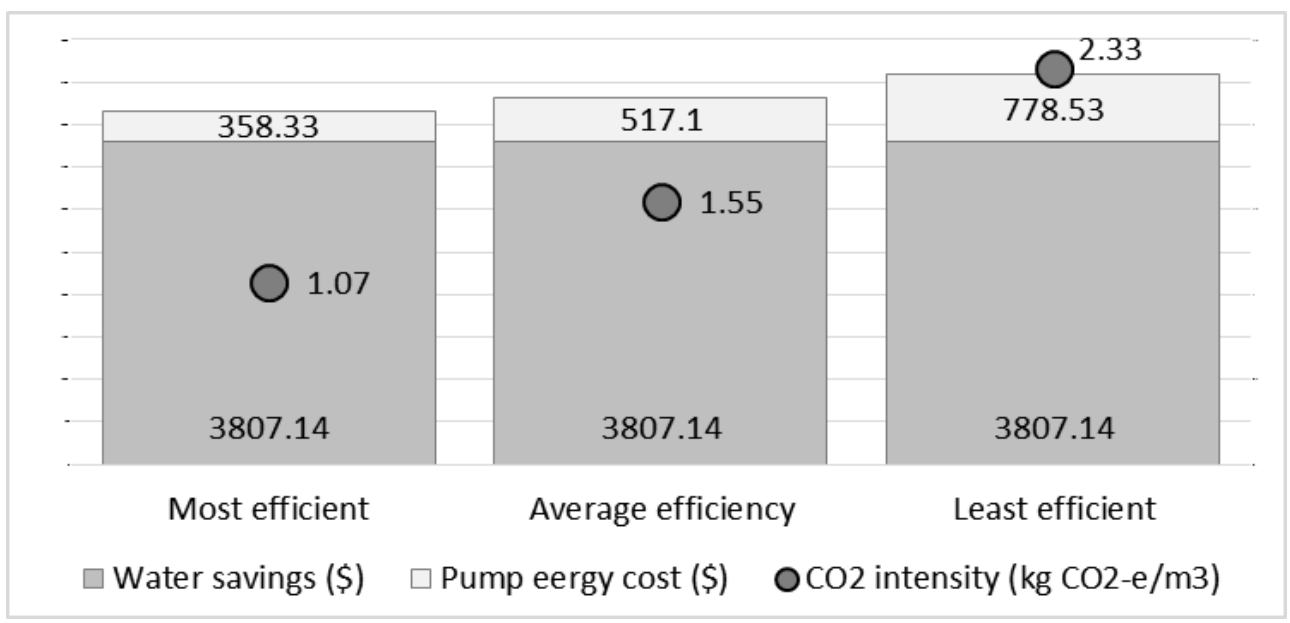

(d) A comparison of 3 scenarios, the average, most efficient and least efficient IPRWTS

Figure 4. High resolution water-energy data collection and analysis 


\subsection{Case study 2: Southern California case study}

\subsubsection{Case study overview}

This case study is synopsis of a recently completed study by the authors (Cominola et al. 2017b). The study illustrates a customer segmentation analysis of residential accounts of water-electricity consumers in Southern California. The authors propose a three-phase customer segmentation analysis developed to (i) discriminate among heterogeneous water-energy use routines (i.e., typical hourly patterns or daily water/energy use) by mining hourly water and electricity use data, (ii) identify groups of consumers to target with management actions aimed at pursuing water/energy conservation and demand peak shifting, and (iii) show policy implications of data mining smart-metered data, by recommending a portfolio of customized demand-side management measures for those targeted users. The use of information from intelligent meters to discover causal and behavioural connections between water and electricity use patterns is key for multi-utilities to cost-effectively design coordinated customized demand management strategies (e.g., tailored consumption feedbacks, pricing schemes, etc.) and, thus, effectively differentiate them for diverse groups of users.

In this case study, the proposed methodology was applied to categorise different water-energy use behaviours for over 1000 residential water and energy consumers, in Burbank (Los Angeles County South California). Each account is described by anonymous hourly water and electricity data, collected by the municipal utility Burbank Water and Power in the second half of 2015 (June - December, 2015). Moreover, each account was characterized by approximately 50 psychographic features collected through an opt-in survey (WaterSmart, 2015), which described occupant demography, household features, personal attitudes especially toward water use and conservation, and stated preferences. In order to extract relevant consumption patterns out of a dataset with over 5 million data points of hourly water-energy use, and cross-correlate it with user psychographics, the authors combined several data mining techniques in the overall customer segmentation approach described below.

\subsubsection{Approach and findings}

The customer segmentation analysis consisted of three main steps. The first is the Eigenbehaviour extraction: this step exploits Principal Component Analysis (PCA) to perform data dimensionality reduction and extract recurring coupled water and electricity daily use patterns, called eigenbehaviours (Eagle and Pentland, 2009; Cominola et al. 2016) from the initial large dataset of hourly water and electricity use data of each account. In this case study, the first eigenbehaviour was found to account for more than $60 \%$ of the total variance of user's consumption data, and so each account was characterized solely by his/her primary eigenbehavior. The second phase is consumer segmentation: water-electricity consumer accounts are clustered based on similarities of the eigenbehaviours assigned in the previous phase. The primary eigenbehaviours of all users are automatically clustered via a 
sequential use of $t$-Distributed Stochastic Neighbor Embedding (van der Maaten, 2008) and K-means clustering.

Clustering the 1107 Burbank's accounts with the above procedure resulted in a set of 18 predominant water-energy eigenbehaviours, i.e., relevant types of water and electricity consumption routines across the whole set of accounts. Finally, factor mapping: in this last step, the 18 predominant water-electricity eigenbehaviors are cross-correlated with the 50 user psychographic features collected via opt-in survey. The Patient Rule Induction Method (Friedman and Fisher, 1999) factor mapping technique was preferred over traditional correlation methods because of the heterogeneous nature of the input features, as well as their uncertainty embedded intrinsically into information declared via surveys. This last phase allowed automatically inferring the most likely determinants of demand drivers for a set of selected topconsuming profiles to target with conservation and demand peak shifting actions.

The outcomes of this study are particularly interesting to inform future digital multi-utilities. First, results demonstrated that the methodology proposed can capture differences and similarities in waterelectricity consumption routines and, thus, extract relevant concise information out of large datasets from intelligent meters. As an example from the mentioned study, Table 3 displays and describes the main heterogeneous predominant eigenbehaviors among the 18 found after the clustering step. In the table, each subplot shows the coefficient of the eigenbehaviours for different levels of water (solid lines) and electricity (dashed lines) consumption: higher coefficients mean higher frequency of a level of consumption for a given hour. The authors of this case study found that the per-household average daily water and electricity uses of Burbank's accounts have a strong linear correlation of 0.93, yet the time of use and hourly patterns are different between water and electricity (as it can be noticed via visual analysis of the profiles in Table 3). These information impacts the definition of criteria utilities and authorities should consider to select target users for their demand management programs, as well as the design of programs based on time of use (e.g., hourly tariffs). First, out of 50 candidate psychographic determinants cross-correlated via factor mapping with primary eigenbehaviours, the main feature automatically identified as a likely driver of large water and electricity demands was the presence of either a swimming pool, a hot tub, or both. Data supported this result, as $75 \%$ of the users belonging to the cluster with the largest water-electricity use own a swimming pool/hot tub/both. Second, the main feature identified as likely demand driver for those top-consumers not owning a swimming pool/hot tub was their medium-to-low sensitivity towards water price and medium-to-low environmental attitude towards water conservation. This result demonstrated that both objective and personal features significantly influence water and electricity use.

Inferring key demand determinants for different segments of consumers would support utilities in designing effective portfolios of customized demand management interventions. Thus, the methodology presented in this case study significantly contributes to behavioural studies on residential 
water-electricity nexus, and its findings relevant implications for coordinated water-energy interventions.

Table 3. Main types of predominant water-energy eigenbehaviors found across Burbank’s accounts.

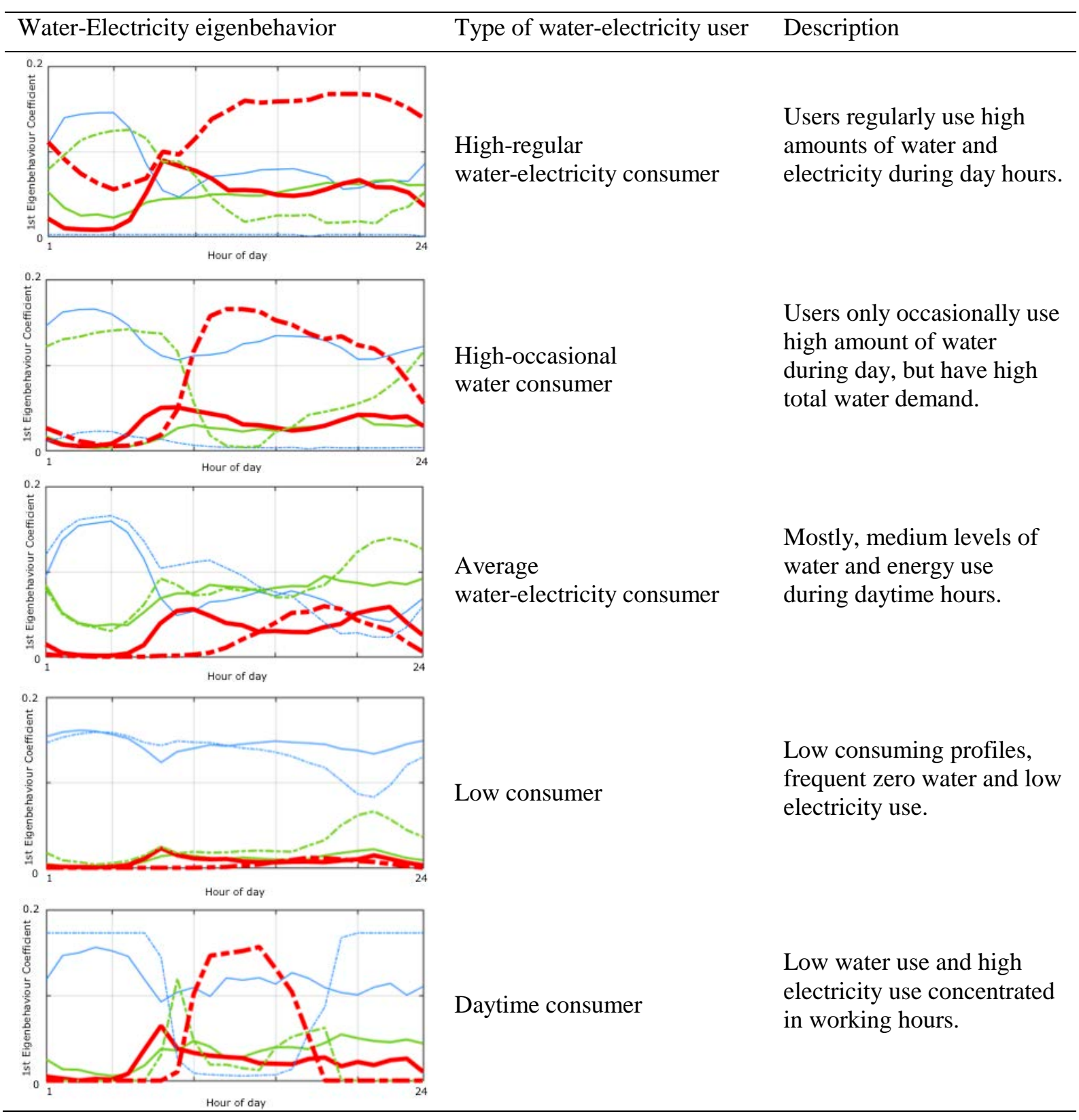

Note: In each subplot: $\mathrm{x}$-axis represents the hour of day, $\mathrm{y}$-axis the coefficient of the $1^{\text {st }}$ eigenbehaviour distinguished among high water use (red solid line), medium water use (green solid line), zero water use (blue solid line), high electricity use (red dashed line), medium electricity use (green dashed line), low electricity use (blue dashed line). Table content was adapted from Cominola et al. (2017b).

\subsection{Case study 3: Household appliance water-energy nexus analysis}

\subsubsection{Case study overview}

This case study presents research conducted collaboratively across the water and energy sectors in Melbourne and Brisbane, Australia. The work aimed to understand water and energy connections in 
seven individual households, and to use this to inform the assessment of water-related energy use, GHG emissions and costs at district scale ( 10,000 households) (Clearwater 2017). The approach involved collection and use of high-resolution water and electricity end-use information across seven widely varying households, to characterise water-related energy. Modelling was undertaken using "ResWE" a mathematical material flow analysis (MMFA) model (Kenway et al. 2013b). Early stages of the project identified that the most significant quantities of water-related energy are generated from shower use (Binks et al. 2016).

\subsubsection{Approach and findings}

Using normalized sensitivity results from the MMFA, the research demonstrated (i) high inter-house variability and (ii) a large and consistent influence of shower duration, flow rate, frequency and temperature along with hot water system efficiency. A $10 \%$ simulated change in these factors influenced $0.1-0.9 \mathrm{kWh} / \mathrm{p} / \mathrm{d}$, equivalent to a $2-3 \%$ of total household energy use. Results from the seven highly characterised households were coupled with behavioural information e.g. duration, flow rate, and frequency, from 5,399 shower events across 94 households, and (much rarer) 491 shower temperature measurements to understand the drivers that could be targeted to reduce current levels of water and energy use, GHG emissions and costs.

Event-based measurements were collected for showers across four households. Amphiro meters (Amphiro 2017) were chosen due to their ability to simultaneously collect temperature, frequency, flowrate and flow duration measurements. When coupled with householder log sheets, the result enabled a high level of partitioning of water-related energy from individual householders: in this case Persons A and B (Figure 5).

Based on the analysis, Person A (in this case a male) was using $0.9 \pm 0.2 \mathrm{kWh}$ energy per shower. And Person B (in this case a female) was using $2.7 \pm 0.7 \mathrm{kWh}$. In the absence of simultaneously compiled information we would not have been able to tease-apart this significant difference in behaviour. 


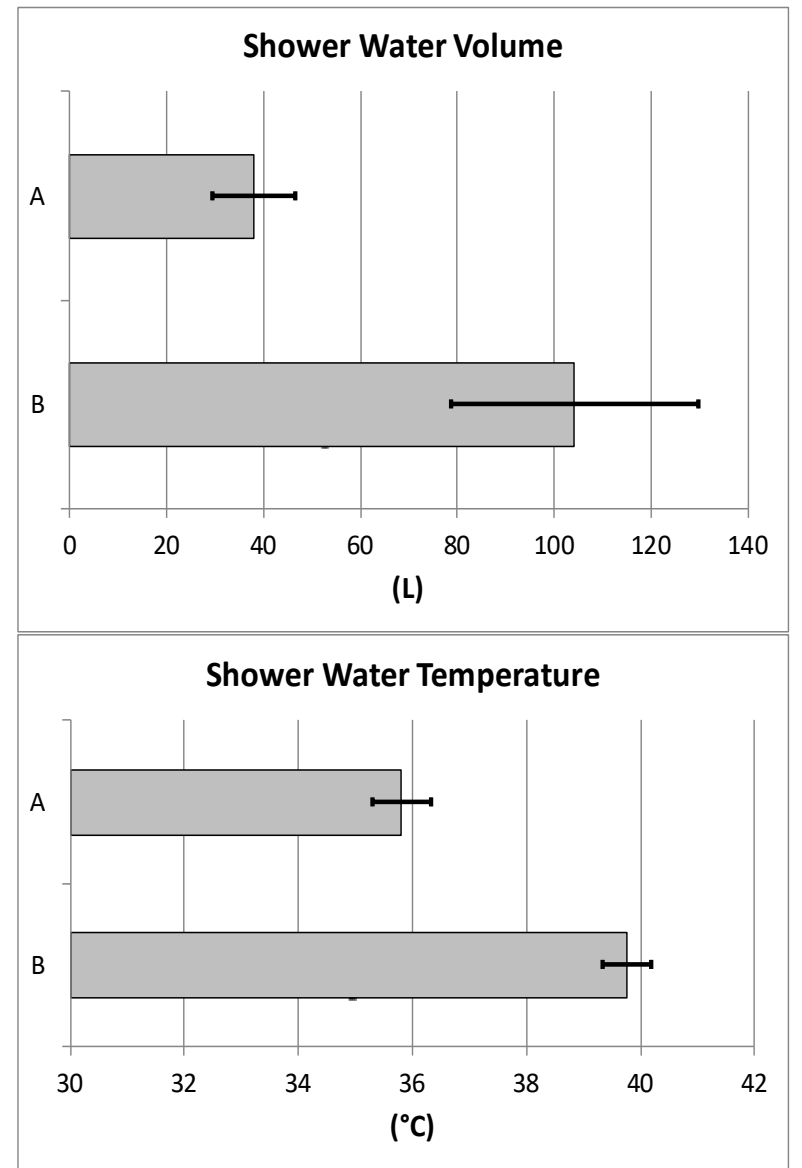

\begin{tabular}{cccc}
\hline Person & $\begin{array}{c}\text { Average } \\
\text { Volume }(\mathrm{L})\end{array}$ & $\begin{array}{c}\text { Avgerage } \\
\text { Temperature }\left({ }^{\circ} \mathrm{C}\right)\end{array}$ & $\begin{array}{c}\text { Sample Size } \\
(\mathrm{N})\end{array}$ \\
\hline $\mathrm{B}$ & 104 & 39.8 & 30 \\
$\mathrm{~A}$ & 38 & 35.8 & 42 \\
\hline Combined & 71 & 37.5 & 72 \\
\hline
\end{tabular}
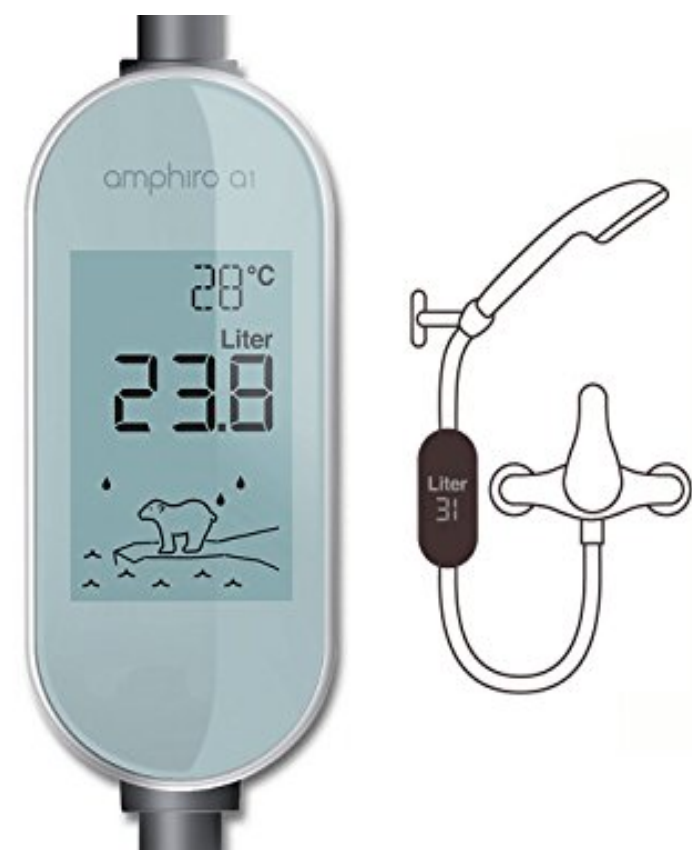

Figure 5. Summary results for volume and temperature for Persons A and B (Amphiro meter).

\subsection{Case study 4: Household multi-utility water-energy ICT system}

\subsubsection{Case study overview}

A multi-utility ICT system that supports the integrated management of urban water demand at both household and utility levels was recently developed in the framework of iWIDGET Project (Savić et al. 2014). The project delivered two innovative cloud-based platforms with advanced data analytics to acquire, transfer, process and visualise information from telemetry systems and smart meters to utility personnel and householders respectively (see Kossieris et al. 2014; Loureiro et al. 2014b; Makropoulos et al. 2014). This case study application is focused on the residential customer platform that enables end-users to monitor and control, on a real-time basis, both water and energy consumption of their household providing valuable information and feedback (Figure 6). More specifically, the main functionalities of the platform included: i) monitoring of total water and energy consumption; ii) coarse breakdown of the total water and energy meter readings into main domestic uses and appliances; iii) detection of unusual consumption events as well as fault events such as bursts and leakages; iv) comparisons/challenges with other households in the monitoring network; v) provision of customised 
suggestions, tips and practices towards water and energy efficiency; and vi) remote control of smart appliances (e.g. dishwasher and washing-machine).

\subsubsection{Approach and findings}

The platform was implemented and validated in three case studies (UK, Portugal and Greece) where data from 15-min up to daily time scales were collected for approximately 2 years. With a focus on the water-energy nexus, smart energy meters were also installed, further to smart water meters, in half of the households of the Athens case study, in Greece. The metering system transferred 15-min water and energy data to a secure storage server which periodically updated the household web 'profile' via the web platform and the user is able to monitor the current energy consumption and the corresponding cost (by specifying the relevant pricing structure) and explore how these are allocated into different time spans (i.e., day/night, summer/winter, working/week-end days' consumption). The system also presents a coarse estimation of the percentage of total energy consumption that is associated with various waterrelated activities (i.e., boiler, dishwasher, washing machine, dryer). At a more detailed level, the platform also estimates and presents the breakdown of total energy consumption into the main energy uses (as specified by the user), also including water-related activities. Since the user is able to specify, within the system, the source of energy (i.e., gas, electricity, solar energy etc.) of the household, the carbon footprint of the household can also be estimated.

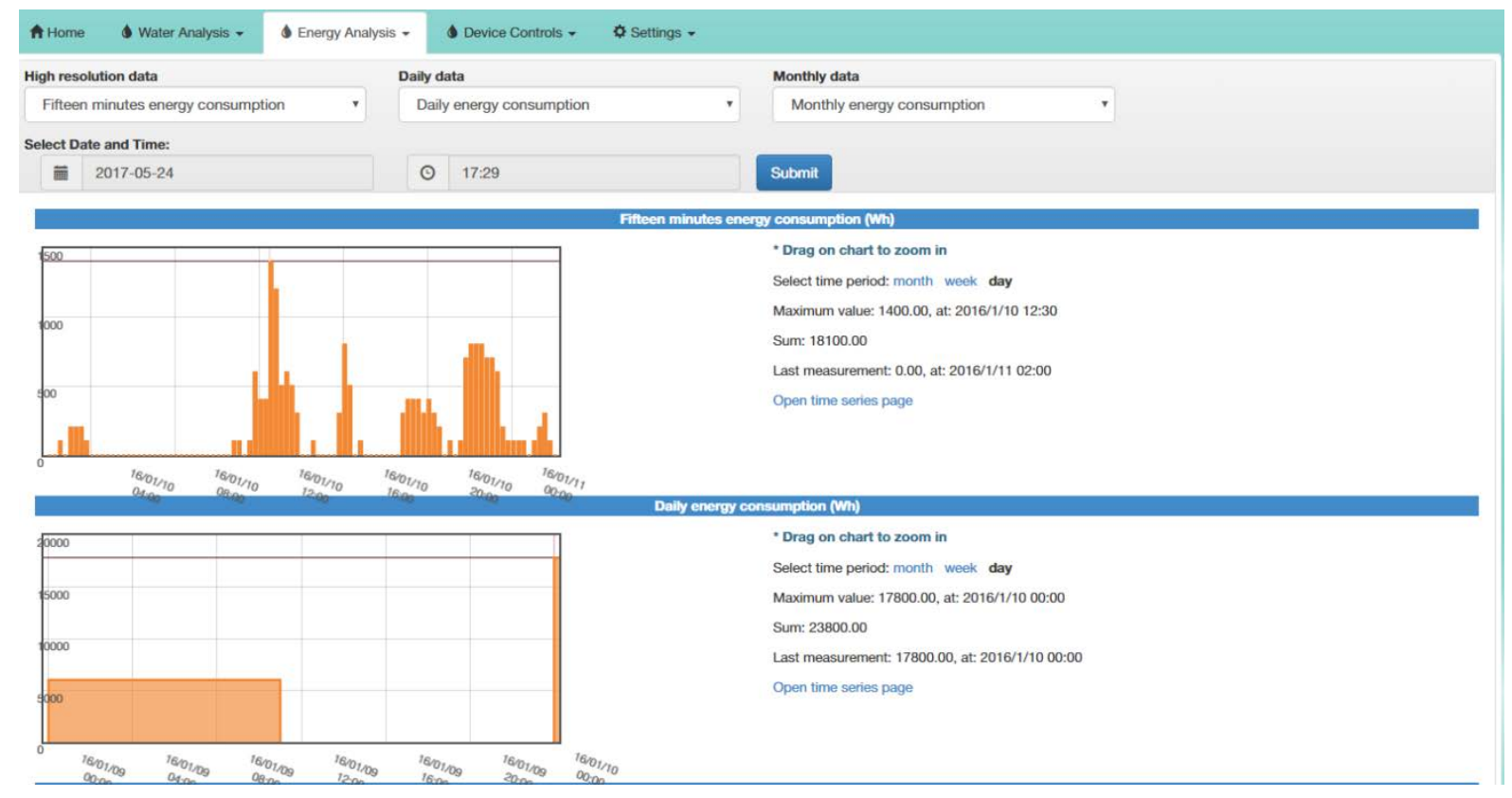

(a) Displays of water related household energy consumption in different timescales (upper: 15 min; lower: hr) 


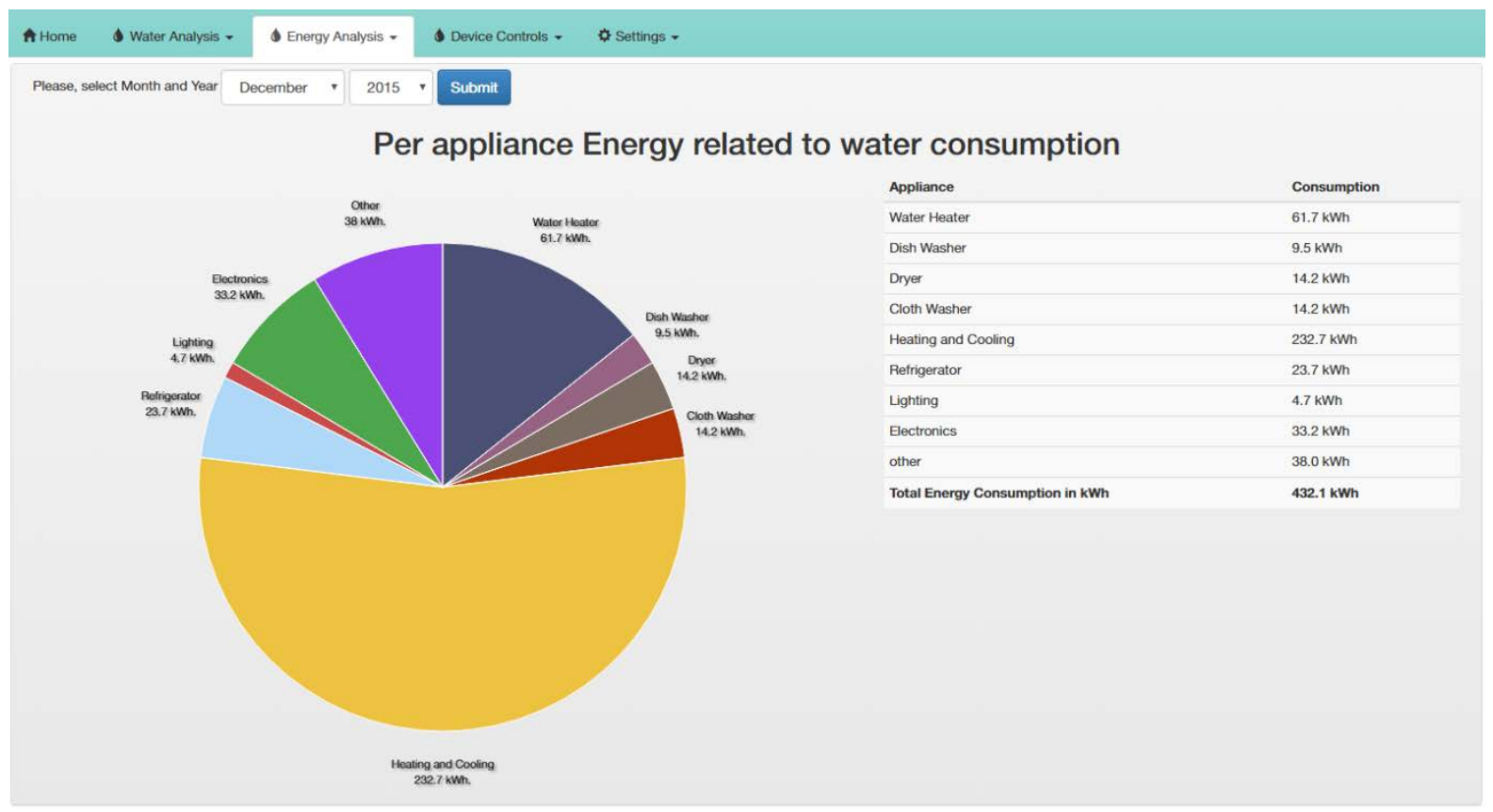

(b) Pie chart and summary table of per appliance energy consumptions also including water-related activities

Figure 6. The iWIDGET household platform for water-energy monitoring and control

\subsection{Case study implications for digital multi-utility}

As demonstrated in the case studies, the digital multi-utility offers a number of new opportunities for both customers and utilities. The use of digital energy and water monitoring technologies, as demonstrated by Case Study 1, would provide multi-utilities and their customers with a real-time direct and important feedback of water and energy consumption and cost. Having high-resolution data is especially important for utilities and customers owning, leasing or managing decentralised water and energy systems (Talebpour et al. 2014; Bennett et al. 2015). In particular, being able to unpack the water-energy nexus of such complex systems is a valuable benefit of digital customer metering. Multiutilities would be able to use data analytics to inform customers having sub-standard systems operations and provide clear advice on how they can be optimised. Concurrently collected multi-utility metering of high-resolution water and electricity data would enable multi-utility providers and customers to reveal a range of system deficiencies at each site.

Advanced customer segmentation of coupled sub-daily water and energy use data, as shown in Case Study 2, would enable multi-utilities to explore heterogeneity and similarities in typical water-electricity demand profiles, identify behavioural nexus and key determinants of target profiles, and design recommendations for joint water-energy demand-side management interventions. This case study demonstrated that water-energy use data metered at a medium (i.e. hourly) sampling resolution contained useful information to characterize consumers' behaviour and habits. Thus, the findings of 
this case study provided vital information on the customers' behaviours in the context of the city-scale residential water-energy nexus and has implications for coordinated water-energy interventions.

The outcome of the household appliance water-energy nexus analysis, shown in Case Study 3, informs the tailoring of behavioural and technological water-efficiency programs towards those with the strongest potential to influence energy. The information also helps guide city-scale analysis of household water-related energy demand. For example, better collection of data on shower water temperature would help improve the resolution and impact of water-related energy savings measures. The results generated from the monitoring, coupled with detailed MMFA modelling across all seven households, has also shown how there is a strong interaction between parameters. This suggests that programs aiming to influence water-related energy need to be aware of how this interplay either amplifies, or diminishes, the intended energy savings.

Case Study 4 presents a comprehensive multi-utility ICT system that provides an integrated platform for urban water demand management at both household and utility level. The platform utilises mediumresolution water and energy data (15-minute interval) to inform customers of their water, water-related energy and energy consumption. Such large case study projects that prototype various features of the future digital multi-utility provider help this emerging industry to understand data types and presentation options that best engage with customers. Such platform would also enable utilities to better analyse the substantial volume of data for their planning needs, improving service quality and customer relationships.

\section{Digital multi-utility transformation $R \& D$ priorities}

A number of research and development (R\&D) activities must be addressed to realise the full applications and benefits of the visioned digital multi-utility service provider (Figure 7). This section briefly outlines the major $R \& D$ priorities to be addressed before the digital multi-utility vision can be realised. These fifteen priorities have been clustered into three broad perspectives, namely, strategy, technology; and information. In addressing the transformational R\&D priorities, it is important to pursue system transformation through a process of co-design with researchers, utilities, technology providers, government and the community and cognisant of current and future practices and preferences in relation to water and energy. 


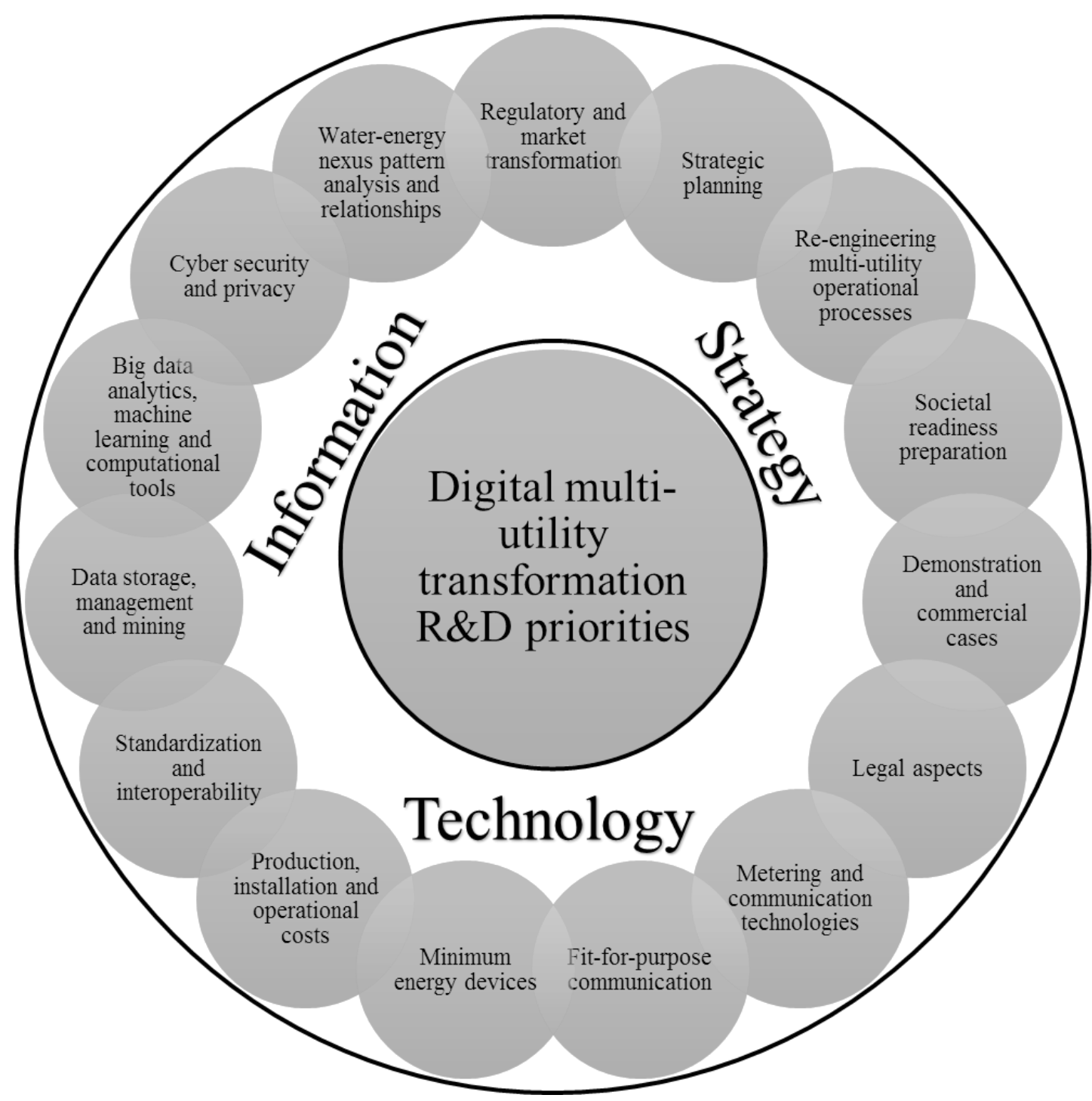

Figure 7. Digital multi-utility transformation R\&D priorities

\subsection{Strategy}

\subsubsection{Regulatory and market transformation}

Research is urgently required to reveal the regulatory and market arrangement hurdles to enable the formation of multi-utility retailers covering services of water, wastewater, energy and gas sectors. This aspect is particularly relevant for the urban water sector where there has been significantly less deregulation occurring than the energy sector. Working towards a policy, regulatory and market environment that supports digital multi-utility transformation is critical. Research is needed to understand the best strategies to foster collaboration between parties and to accelerate the diffusion of demonstration and deployment projects. 


\subsubsection{Digital multi-utility transformation strategic planning}

Digital metering deployment requires meticulous strategic planning (Zhou and Brown, 2017). Repositioning energy and water utility sectors with long established traditions, to include space for digital multi-utility service providers, will be challenging and will require successful strategic planning, pilot and citywide rollouts, technology push, and customer pull. Strategic management research is needed in order to understand the best mechanisms and frameworks to streamline this transformative agenda with minimal risk and maximised value adding to customers and the utility sector business stakeholders (i.e. generators/suppliers, distributors, etc.).

\subsubsection{Re-engineering multi-utility operational processes}

Management consultants and technology firms will often spruik the benefits of their disruptive technology to utilities without a complete understanding of the operational processes of the utility. Failed technology projects are often related to inadequate strategic planning of the tasks that need to be re-engineered (Stewart et al. 2002; Stewart 2008). Digital multi-utility transformation requires a complete understanding of the traditional and potential re-engineered processes of a utility. Big data is only value adding when it provides synthesised information to an end-user for more productive operational processing or decision making. Therefore, research is required to complete work studies on existing processes, and then vision and comparatively analyse identified key performance indicators for re-engineered digital multi-utility processes. After such work studies and re-engineering assessments are completed, a strategic digital multi-utility transformation plan can be developed and implemented in a well-planned manner that is likely to be realise all envisaged benefits.

\subsubsection{Societal readiness preparation}

Utilities are consumed by various customers, including commercial, industrial and residential. Within these segments, there are various sub-segments each having particular needs and wants for their utility consumption data. Proactive societal readiness planning is particularly important counteract the vocal opponents to digital utility technologies (e.g. Stop smart meter groups), which have made traditional utilities nervous about such technologies and delayed their widespread rollout (Boyle et al. 2013). Efforts to demonstrate that digital multi-utilities will not adversely affect vulnerable consumer groups and endanger privacy rights through information sharing with authorities is especially required (Giurco et al. 2010). Often, a lack of educating the public on the benefits of digital technologies, examples of failed digital systems, and utilities failing to demonstrate that transformative changes will yield cost benefits for customers prior to a rollout, has given opponents adequate ammunition to create fearful campaigns on the potential negative risks of this technology. Socio-behavioral research is needed to firstly understand different customer segments requirements, wants and concerns related to digital 
multi-utility solutions and then to secondly formulate technology diffusion strategies that address these acceptance factors.

\subsubsection{Demonstration and commercial cases of digital multi-utility applications}

Many early adopters of innovative technologies have failed to realize the intended transformative benefits promised by their technology consultants (Panuwatwanich and Stewart, 2012). Reported cases of failed technology programs that have failed to meet hyped expectations make others firms reluctant to be technology leaders. This reluctance to lead the technology agenda is prevalent in the utility sector which is characterized by conservative practices and slow change (Stewart et al. 2010). An R\&D strategy to facilitate digital multi-utility transformation is needed to conduct increasingly scaled pilot, demonstration and eventually commercial sized program implementation. These 'real life' pilot and demonstration projects will mitigate later implementation risks associated with a larger rollout, by resolving issues (e.g. technology, environment, change management, etc.) at an early stage.

\subsubsection{Legal aspects}

There are a number of yet to be resolved debates on the privacy, rights and access legal implications of extensive databases containing interval data on a customers, water, energy and gas demand, that a digital multi-utility providers will be a custodian over (Brown 2013). Advanced pattern recognition algorithms has the potential to unpack the end use activities of customers (e.g. showering, making a cup of tea, etc.) at a particular time of the day (Gurung et al. 2016; Beal et al. 2016b). Moreover, the digital multiutility having access to interval data may also have a heightened legal responsibility (i.e. duty of care) to act on certain issues reported in a timely manner. For example, if continuous water usage is identified by the digital meter, the utility will have a duty to immediately inform customers of a potential leak (Britton et al. 2013). A research program is required that proactively addresses the many legal implications of digital multi-utility transformation.

\subsection{Technology}

\subsubsection{Fit-for-purpose communication systems}

Determining the most appropriate communications systems for digital multi-utility companies for different customer segments and locations is required. Customer properties may be located in densely or sparsely populated areas having different available ICT infrastructure. For different situational context and available infrastructure, fit-for-purpose and reliable communications systems are needed. Whether using private utility communication networks (i.e. radio networks, mesh networks, etc.) or public carriers and networks (Internet, cellular, cable or telephone), the communication network should be able to transfer adequate multi-utility data simultaneously to the utility provider. While a number of 
studies and demonstration projects have detailed particular architectures for different single utility provider scenarios (Kabalci, 2016), further research is required to validate that these approaches and to extend them by meeting the requirements for multi-utility data transfer.

\subsubsection{Digital multi-utility metering and communications technologies}

Concentrated R\&D activities are required to improve the current product range of digital multi-utility metering and communication technologies. Improvements are required in product resilience to harsh outdoor conditions (e.g. meters are often external to properties and exposed to environmental conditions and vandalism), data interval resolution recording (i.e. second data), data storage availability, communications reliability and security, to name a few. Customers will only have faith in digital multiutility technologies when they have confidence that these technologies will consistently provide them with useful accurate data over the long term. Detailed technical specifications for such technologies that will ensure the realisation of the vision of a digital multi-utility need to be formulated and provided to industry product manufacturers and technology providers.

\subsubsection{Designing minimum energy devices}

Water and gas meters are often removed from a continuous power source, making them reliant on longlife battery sources. Such digital meters will need to be reliant on their own limited energy resources or some innovative remote energy harvesting inclusion throughout their lifetime. Low power communications are receiving considerable research attention, with IEEE standard, Bluetooth, ultrawide bandwidth, and RFID/NFC technologies all working towards low energy solutions (Yaboob et al. 2017). In addition to reducing digital meter power consumption, there are potential research opportunities to explore energy harvesting opportunities (Ma et al. 2016; Villani et al. 2016). For example, for water meters, there are examples of harvesting water flow to harvest energy to sustain digital water meter requirements (Kroener et al. 2016). Undoubtedly, power efficiency and harvesting are an ongoing $R \& D$ agenda for the realization of the digital multi-utility provider.

\subsubsection{Digital multi-utility system production, installation and operational costs}

A core driver or impediment of digital multi-utility transformation is the cost-benefit equation. While technology and operational costs remain high, the benefits will be outweighed by the costs. Only through technology commercialisation and mass market production along with a utility sector becoming experienced with installing and operating sophisticated multi-utility systems, will costs reduce to a point where technology becomes the driver of transformation. R\&D funding is needed internationally to reduce the costs of digital metering technologies, and to complete pilot and demonstration projects to demonstrate its fitness-for-purpose for widespread implementation. Collaborative partnerships between Universities, Research Institutes, technology providers and utilities is essential, to conduct research and 
development activities, as well as to train a new generation of utility professionals that are savvy with digital technologies and informatics.

\subsubsection{Standardization and interoperability}

Ideally, the various advanced water, electricity and gas metering systems available have sufficient compatibility to work together in order to logically and efficiently provide their data to a multi-utility service providers. However, such desired standardization and interoperability has not been realised in the utility sector, requiring an urgent push for a framework and research program addressing the four main areas for interoperability, namely: (1) technical interoperability; (2) syntactic interoperability; (3) semantic interoperability; and (4) organizational interoperability. Different technologies and components involved in data processing still use proprietary or ad-hoc protocols or data formats, making the exchange of data among different systems or the interconnection of components for a combined processing of the information complex.

\subsection{Information}

\subsubsection{Data storage, management and mining}

Data management is a critical requirement for creating digital multi-utilities where a number of interconnected metering devices are constantly exchanging all types of information, the sheer volume of the generated data and the processes involved in the handling of such data is of paramount importance. The potential volume of multi-utility data being collected and automatically stored in information systems will be huge and storage servers must be capable of storing this data. Moreover, stored data must be pre-processed to ensure reliability (i.e. missing data, erroneous data, etc.), and stored logically for subsequent processing and extraction. Significant research effort is needed to define and implement semantics and rules for streamlining information processing.

Data representation and mining algorithms should be capable of handling higher levels of abstraction and information manipulation, and allow for the subsequent employment of more complex pattern recognition and informatics algorithms that can handle complex interrelationships between multiple utility data sources (e.g. determining the water-related energy required for a clothes washer event).

\subsubsection{Big data analytics, machine learning and computational tools}

Intelligent digital multi-utility meters will produce massive datasets that have limited value unless they can be analysed to unpack a number of trends. Brunswicker et al. (2015) recently provided a description of the research activities related to 'big data' analytic tools and computational techniques. They identified that research is need in the following six areas: (1) Meta-network modelling; (2) Network discovery and network analysis methodologies; (3) Dynamic network analysis and statistical prediction; 
(4) Agent-based simulation models; (5) Behavioural sequencing techniques and genetic computation; and (6) Collaborative and automated coding tools for unstructured text data.

\subsubsection{Cyber-security and privacy}

The digital multi-metering system will need to be able to send very detailed demand information about customers' water, wastewater, electricity and gas usage, which can be assessed both legitimately and illegitimately (McHenry, 2013) and also unintentionally (Yan et al. 2013). Researchers and standards organizations are working on secure data transfer protocol technologies for both wireless (e.g. IEEE 802.16e) and wired connections (e.g. SSH/SSL) (Ahmad et al. 2016); however, hackers repeatedly come up with means to circumvent these technologies requiring further research and enhancements.

Research is required into attack detection, vulnerability metrics and recovery/resilience for digital multi-utility specific threats, as well as management response decision support procedures and tools. Privacy, authentication and data ownership in the context of globally distributed digital multi-utility systems is another key area of research. Ideally, the design of voluntary "opt-in” paradigms may be needed for enabling some features of the digital multi-utility system. To alleviate privacy concerns that customers may have related to digital multi-utility transformation, there is a requirement for research to support anonymity and restrictive handling of utility customer information.

Research is required in cryptographic techniques that restrict information content being accessible to other parties, designing more edge computing and processing applications, methods to support Privacy by Design approaches, including data minimization, identification, authentication and anonymity (Brown, 2013), and research underpinning techniques allowing for the greater use of soft identities (Nadargi, and Thirugnanam, 2016).

\subsubsection{Water-energy nexus pattern analysis and relationships}

The realisation that water-related energy is a significant proportion of total energy demand has prompted researchers to analyse collected data to reveal efficiency opportunities (Beal et al. 2012; Beal et al. 2016). Moreover, while there is some anecdotal understanding of the relationship between customer water and energy demand, there is still a lack of evidence-based research supporting certain water-energy nexus trends. Detailed understanding has not been possible because until recently there has been limited high resolution water and energy data available, especially concurrently occurring energy (electricity and/or gas) and water data that can be directly correlated to reveal interesting waterenergy nexus trends. Digital multi-utility pilot and demonstration projects will deliver significant datasets that could underpin data mining and pattern recognition research studies to reveal a range of customer water, electricity and gas demand trends, as well as the more complex water-energy nexus trends. 


\section{Conclusion}

While the rate of diffusion has been slower than initial expectations, it is inevitable that individual utility organisations will embrace digital technologies to more efficiently and effectively manage their assets, while significantly enhancing their level of engagement with customers. Technology companies such as Google, Uber, Facebook, and others, have shown that new data types and/or abundant existing data and/or aggregating/synthesising available data creates new business opportunities for exploitation. Digital and AI disruption can be delayed by a particular industry sector but not avoided in the longer term. The leading digital multi-utility provider has the opportunity to not only extract numerous benefits from the new and abundant data from intelligent metering and monitoring of an individual utility sector (e.g. water sector), but also from the aggregation of concurrent multi-utility datasets (i.e. coupling water, electricity and gas demand data).

While there are a number of technical and management studies demonstrating the applications and benefits of mining and modelling big data from smart or intelligent single utility networks, there are few that showcase the aggregation opportunities presented by the future digital multi-utility network. From a modelling and software viewpoint, the combined concurrently collected water, electricity and gas information of each and every customer, enables greater accuracy and granularity of pattern recognition as well as significantly enhanced understanding of each utilities demand. There is a dearth of studies completed by data informatics and modelling researchers that have unlocked the necessary techniques and tools for this emerging sector to be able to effectively harness combined digital multiutility sector datasets. A new research field of multi-utility resource informatics will emerge.

This paper has demonstrated examples and showcased recent reported pilot cases where digital multiutility data has been analysed for a particular application. The purpose of showing and explaining these illustrative examples is to seed novel thought and motivate greater R\&D attention to this field, in order to formulate the techniques and tools required to extract the full suite of herein explained customer and utility benefits of this transformative agenda. While the heart of the paper is focused on the technical data mining and modelling challenges and opportunities related to the formation of a digital multiutility, which is in line with the scope and readership of this journal, the paper also purposely includes the overarching $R \& D$ agenda for this transition. This $R \& D$ priorities section was included to acknowledge that the data mining and modelling tasks are just a few of the priorities that must be addressed in order to facilitate digital multi-utility transformation. The described technology, strategy and information categories of priorities must all receive $\mathrm{R} \& \mathrm{D}$ attention before the visioned digital multiutility service provider can be realised. 


\section{Acknowledgements}

For Case Study 1, Prof. Stewart and Dr. Sahin acknowledge the work of Ray Siems from Cambridge University and Reza Talebpour from Griffith University. Project support is acknowledged from the Australian Research Council (ARC) Linkage Project (LP100200033) that also includes funding from the industry partner Gold Coast City Council. The University of Technology (UTS), Sydney and CSIRO are also partnered investigators to this grant. Special thanks to Griffith eResearch Services Team for developing and supporting the Smart Meter Information Portal, and Dr Ruby Gonzales at Griffith University for establishing an FTP server. Finally, the authors greatly appreciate the kind support of the households involved in the study.

For Case Study 2, Prof. Castelletti, Dr. Cominola, and Dr. Giuliani acknowledge the work of Prof. Edward Spang, Prof. Jay Lund, and Prof. Frank Loge in supporting the research leading to these results and in co-authoring the paper from which Case Study 2 was adapted. The authors would like to acknowledge the Centre for Water and Energy Efficiency (UC Davis) and Burbank Water and Power for sharing the anonymized data used in this research. This research has received funding from the European Union's Seventh Framework Programme (FP7/2007-2013) under grant agreement no. 619172 (SmartH2O: an ICT Platform to leverage on Social Computing for the efficient management of Water Consumption; http://smarth2o.deib.polimi.it/).

For Case Study 3, A/Prof. Kenway acknowledges the work of Amanda Binks (installation of Amphiro Meters and MMFA modelling), Verena Tiefenbeck (ETH Zurich for processing of Amphiro raw data) and Kristy Fox (assessment of processed Amphiro data). A/Prof Kenway also acknowledges the support of the Australian Research Council (DECRA Project DE 160101322) in the preparation of this paper.

For Case Study 4, Prof. Savic, A/Prof. Makropoulos and Mr Kossieris acknowledges the iWIDGET (http://www.i-widget.eu/) research team and industry partners. The authors also acknowledge the funding provided European Union's Seventh Framework Program for research, technological development and demonstration under grant agreement no. 318272.

\section{Software/data availability}

Section reference: Case Study 1

Name of software: Autoflow

Developer: Dr. Khoi Nguyen

\section{Contact information:}

Dr. Khoi Nguyen

Research Fellow

Griffith School of Engineering 
Griffith University

Ph: +61 755527522

Email: k.nguyen@griffith.edu.au

First year available: 2015

Program language: MATLAB

Software availability: Restricted

Software size: $44 \mathrm{MB}$

Software required: MATLAB Compiler Runtime (MCR)

Hardware required: $2.4 \mathrm{GHz}$ processor and 2GB RAM

Dataset:

Location: Melbourne and Southeast Queensland - Australia

Size: 500 residential households

Time: 2-week period in 2010, 2011 and 2014

http://gc-prd-ersservices.rcs.griffith.edu.au/smip2/

Dataset Availability: restricted

Reference articles DOI:

https://doi.org/10.1016/j.asoc.2015.03.007

https://doi.org/10.1016/j.jher.2013.02.004

https://doi.org/10.1016/j.envsoft.2013.05.002

https://doi.org/10.1016/j.eswa.2013.07.049

Developer: Dr. Andrea Cominola

Contact information:

Dr. Andrea Cominola

Postdoctoral research fellow

Politecnico di Milano

Email: andrea.cominola@polimi.it

Website: http://www.nrm.deib.polimi.it/?page_id=35

First year available: 2017

Program language: MATLAB (tested with Matlab R2016a), Python (tested with version 2.7.8)

Hardware required: tested on a $2.5 \mathrm{GHz}$ Intel Core i5 processor and 4GB RAM machine.

Software availability: Restricted

Dataset:

Location: City of Burbank - California (USA)

Size: 1107 residential accounts

Time: June $28^{\text {th }}-$ December $8^{\text {th }}$, 2015

Dataset Availability: restricted 
Funding project name: SmartH2O

Project web site: http://www.smarth2o-fp7.eu/

Contact information: smarth2o.deib.polimi.it/contact/

Publication, software and data information:

smarth2o.deib.polimi.it/results/deliverables/

smarth2o.deib.polimi.it/results/publications/

smarth2o.deib.polimi.it/results/software/

smarth2o.deib.polimi.it/results/datasets/

Section reference: Case Study 3

Name of software: Residential Water Energy Model (RESwe)

Developer: Dr. Steven Kenway, Hans Peter Bader, Ruth Scheidegger

\section{Contact information:}

Associate Professor Steven Kenway

Research Group Leader, Water-Energy-Carbon

School of Chemical Engineering,

Postal Address: Level 3, Chemical Engineering Building (74)

University of Queensland, St Lucia, 4072 Australia

s.kenway@uq.edu.au

First year available: 2015

Program language: HTBASIC and SIMBOX (Eawag)

Software availability: Restricted

Software size: $2 \mathrm{MB}$

Software required: HTBASIC and SIMBOX

Hardware required: $2.4 \mathrm{GHz}$ processor and 2GB RAM

Dataset:

Location: Melbourne and Brisbane - Australia

Dataset Availability: restricted

\section{Reference articles DOI:}

http://www.sciencedirect.com/science/article/pii/S0378778816308088

http://www.sciencedirect.com/science/article/pii/S0959652616307685

Paper section reference: Case Study 4

Name of software: iWIDGET

Project web site: $w w w . i-w i d g e t . e u$ 
Contact information: www.i-widget.eu/contacts.html

Publication and software information: www.i-widget.eu/publications.html

\section{References}

Ahmad, M. W., Mourshed, M., Mundow, D., Sisinni, M., \& Rezgui, Y. (2016). Building energy metering and environmental monitoring - A state-of-the-art review and directions for future research. Energy and Buildings, 120, 85-102

Albert, A., \& Rajagopal, R. (2013). Smart meter driven segmentation: What your consumption says about you. IEEE Transactions on power systems, 28(4), 4019-4030.

Al-Rousan, M., \& Al-Ali, A. (2006). An innovative m-utility management and billing system. Journal of Enterprise Information Management, 19(2), 136-147.

Amphiro (2017) Save energy while showering. Amphiro (ed), Zurich.

Anderson, K. (2014). Non-Intrusive Load Monitoring: Disaggregation of Energy by Unsupervised Power Consumption Clustering (Doctor of Philosophy thesis, Carnegie Mellon University, Pittsburgh, PA, USA).

Armel, K. C., Gupta, A., Shrimali, G., \& Albert, A. (2013). Is disaggregation the holy grail of energy efficiency? The case of electricity. Energy Policy, 52, 213-34.

Australian Bureau of Statistics (2013). Environmental Issues: Water use and Conservation, Canberra, Australia. Available: http://www.abs.gov.au/ausstats/abs@.nsf/mf/4602.0.55.003/

Bahmanyar, A., Jamali, S., Estebsari, A., Pons, E., Bompard, E., Patti, E., \& Acquaviva, A. (2016). Emerging smart meters in electrical distribution systems: Opportunities and challenges. Proceedings of the 24th Iranian Conference on Electrical Engineering (ICEE 2016), pp. 1082-1087, Shiraz, Iran.

Beal, C. D., Stewart, R.A., \& Bertone, E. (2012). Evaluating the energy and carbon reductions resulting from resource-efficient household stock. Energy and Buildings, 55, 422-432.

Beal, C. D., \& Flynn, J. (2015). Toward the digital water age: Survey and case studies of Australian water utility smart-metering programs. Utilities Policy, 32, 29-37.

Beal, C. D., \& Stewart, R. A. (2011). South East Queensland Residential End Use Study: Final Report. Urban Water Security Research Alliance Technical Report No 47.

Beal, C. D., Gurung, T. R., \& Stewart, R. A. (2016a). Demand-side management for supply-side efficiency: Modeling tailored strategies for reducing peak residential water demand. Sustainable Production and Consumption, 6, 1-11.

Beal, C. D., Gurung, T. R., \& Stewart, R. A. (2016b). Modelling the impacts of water efficient technologies on energy intensive water systems in remote and isolated communities. Clean Technologies and Environmental Policy, 18(6), 1713-1723. 
Beckel, C., Sadamori, L., Staake, T., \& Santini, S. (2014). Revealing household characteristics from smart meter data. Energy, 78, 397-410.

Bennett, C. J. Stewart, R. A., \& Lu, J. W. (2015). Development of a three-phase battery energy storage scheduling and operation system for low voltage distribution networks. Applied Energy, 146, $122-$ 134.

Bennett, J. B., Stewart, R. A., \& Lu, J. W. (2014). Forecasting low voltage distribution network demand profiles using a pattern recognition based expert system. Energy, 67, 200-212.

Binks, A. N., Kenway, S. J., Lant, P. A., \& Head, B. W. (2016). Understanding Australian household water-related energy use and identifying physical and human characteristics of major end uses. Journal of Cleaner Production 135, 892-906.

Boyle, T., Giurco, D., Mukheibir, P., Liu, A., Moy, C., White, S., \& Stewart, R. (2013). Intelligent Metering for Urban Water: A Review. Water, 5(3), 1052-1081.

Britton, T. C., Stewart, R. A., \& O'Halloran, K. R. (2013). Smart metering: enabler for rapid and effective post meter leakage identification and water loss management. Journal of Cleaner Production, 54, 166-176.

Brown, I. (2013). Britain's smart meter programme: A case study in privacy by design. International review of law, computers and technology, 28(2), 172-184.

Brunswicker, S., Bertino, E., \& Matei, S. (2015). Big data for open digital innovation - A research roadmap. Big Data Research, 2(2), 53-58.

Bulkeley, H., McGuirk, P. M., \& Dowling, R. (2016). Making a smart city for the smart grid? The urban material politics of actualising smart electricity networks. Environment and Planning A, 48(9), 1709-1726.

Cardell-Oliver, R., Wang, J., \& Gigney, H. (2016). Smart meter analytics to pinpoint opportunities for reducing household water use. Journal of Water Resources Planning and Management, 142(6), 04016007.

Castelnuovo, M., \& Fumagalli, E. (2013). An assessment of the Italian smart gas metering program. Energy Policy, 60, 714-721.

Cheong, S. M., Choi, G. W., \& Lee, H. S. (2016). Barriers and Solutions to Smart Water Grid Development. Environmental Management, 57, 509-515.

Clearwater (2017). Water-Energy-Carbon Links in Households and Cities: a New Paradigm, Victorian State Government and Melbourne Water. Melbourne.

Cleveland, F. M. (2008). Cyber security issues for advanced metering infrastructure (AMI). Power and Energy Society General Meeting-Conversion and Delivery of Electrical Energy in the 21st Century, IEEE, pp. 1-5, Pittsburgh, PA, USA.

Cominola, A., Giuliani, M., Piga, D., Castelletti, A., \& Rizzoli, A. E. (2015). Benefits and challenges of using smart meters for advancing residential water demand modeling and management: A review. Environmental Modelling \& Software, 72, 198-214. 
Cominola, A., Giuliani, M., Piga, D., Castelletti, A., \& Rizzoli, A. (2017a). A hybrid signature-based iterative disaggregation algorithm for non-intrusive load monitoring. Applied Energy, 185, 331-344.

Cominola, A., Giuliani, M., Piga, D., Castelletti, A., \& Rizzoli, A. (2017b). Segmentation analysis of residential water-electricity demand for customized demand-side management programs. Journal of Cleaner Production (Under review).

Cominola, A., Moro, A., Riva, L., Giuliani, M., \& Castelleti, A. (2016). Profiling residential water users' routines by eigenbehavior modelling. Proceedings of the International Congress on Environmental Modelling and Software, Toulouse, France.

Conrad, S., Kenway, S. J., \& Jawad, M. (2017). Water and Electric Utility Integrated Planning. Foundation, W.R. (ed), Denver.

Creaco, E., Kossieris, P., Vamvakeridou-Lyroudia, L., Makropoulos, C., Kapelan, Z., \& Savic, D. (2016). Parameterizing residential water demand pulse models through smart meter readings. Environmental Modelling \& Software, 80, 33-40.

Darby, S. (2010). Smart metering: what potential for householder engagement? Building Research \& Information, 38(5), 442-457.

Dedrick, J., \& Zheng, Y. (2011). Smart grid adoption: a strategic institutional perspective. Proceedings of the Annual Conference, Industry Studies Association, Pittsburgh, PA, USA.

Depuru, S. S. S. R., Wang, L., \& Devabhaktuni, V. (2011). Smart meters for power grid: Challenges, issues, advantages and status. Renewable and Sustainable Energy Reviews, 15(6), 2736-2742.

Eagle, N, \& Pentland, A. (2009). Eigenbehaviors: identifying structure in routine. Behavioral Ecology and Sociobiology, 63, 1057-1066.

Ehrhardt-Martinez, K., Donnelly, K. A., \& Laitner, J. A. (2010). Advanced Metering Initiatives and Residential Feedback Programs: A Meta-Review for Household Electricity-Saving Opportunities. Technical Reports E105, American Council for an Energy-Efficient Economy, Washington, DC, USA.

El-Hawary, M. E. (2014). The smart grid - State-of-the-art and future trends. Electric Power Components and Systems, 42, 239-250.

Environment Agency (2010). Harvesting rainwater for domestic uses: an information guide, UK. Available: $\quad$ http://webarchive.nationalarchives.gov.uk/20140328084622/http:/cdn.environmentagency.gov.uk/geho1110bten-e-e.pdf

Erevelles, S., Fukawa, N., \& Swayne, L. (2016). Big Data consumer analytics and the transformation of marketing. Journal of Business Research, 69(2), 897-904.

Escriva-Bou, A., Lund, J. R., \& Pulido-Velazquez, M. (2015). Modeling residential water and related energy, carbon footprint and costs in California. Environmental Science \& Policy, 50, 270-281.

Estache, A., Gomez-Lobo, A., \& Leipziger, D. (2001). Utilities privatization and the poor: lessons and evidence from Latin America. World Development, 29(7), 1179-1198. 
Fagiani, M., Squartini, S., Gabrielli, L., Severini, M., \& Piazza, F. (2016). A Statistical Framework for Automatic Leakage Detection in Smart Water and Gas Grids. Energies, 9, 665.

Fang, X., Misra, S., Xue, G., \& Yang, D. (2012). Smart grid - the new and improved power grid: A survey. IEEE communications surveys \& tutorials, 14(4), 944-980.

Faruqui, A., Sergici, S., \& Sharif, A. (2010). The impact of informational feedback on energy consumption - a survey of the experimental evidence. Energy, 35(4), 1598-1608.

Fidar, A., Memon, F. A., \& Butler, D. (2010). Environmental implications of water efficient microcomponents in residential buildings. Science of the Total Environment, 408(23), 5828-5835.

Fielding, K. S., Spinks, A., Russell, S., McCrea, R., Stewart, R. A., \& Gardner, J. (2013). An experimental test of voluntary strategies to promote urban water demand management. Journal of Environmental Management, 114, 343-351.

Fischer, C. (2008). Feedback on household electricity consumption: a tool for saving energy? Energy Efficiency, 1(1), 79-104.

Friedman, J.H., Fisher, N.I., (1999). Bump hunting in high-dimensional data. Statistics and Computing 9, 123-143.

Gadekar, G. B., \& Chandgude, C.P., (2017). Blockchain and its applications. International Journal of Recent Trends in Engineering and Research 3, 143-145.

Graffy, E., \& Kihm, S. (2014). Does disruptive competition mean a death spiral for electric utilities. Energy Law Journal, 35, 1.

Gaiser, K., \& Stroeve, P. (2014). The impact of scheduling appliances and rate structure on bill savings for net-zero energy communities: application to West Village. Applied Energy, 113, 1586-1595.

Galbraith, K. (2012). Saving up for a dry day, The New York Times, New York. Available: http://www.nytimes.com/2012/11/15/business/energy-environment/15iht-green15.html

Gans, W., Alberini, A., \& Longo, A. (2013). Smart meter devices and the effect of feedback on residential electricity consumption: Evidence from a natural experiment in Northern Ireland. Energy Economics, 36, 729-743.

Geller, H., Harrington, P., Rosenfeld, A. H., Tanishima, S., Unander, F. (2006). Polices for increasing energy efficiency: thirty years of experience in OECD countries. Energy Policy, 34(5), 556-73.

Giurco, D., Carrard, N., McFallan, S., Nalbantoglu, M., Inman, M., Thornton, N., \& White, S. (2008). Residential end-use measurement guidebook: a guide to study design, sampling and technology. Prepared by the Institute for Sustainable Futures, UTS and CSIRO for the Smart Water Fund, Victoria.

Giurco, D., White, S., \& Stewart, R. (2010). Smart metering and water end-use data: Conservation benefits and privacy risks. Water, 2(3), 461-467.

Gungor, V. C., Sahin, D., Kocak, T., Ergut, S., Buccella, C., Cecati, C., \& Hancke, G.P. (2011). Smart grid technologies: Communication technologies and standards. IEEE Transactions on Industrial informatics, 7, 529-539. 
Gurung, T. R., Stewart, R. A., Beal, C. D., \& Sharma, A. K. (2016). Smart meter enabled informatics for economically efficient diversified water supply infrastructure planning. Journal of Cleaner Production, 135, 1023-1033.

Gurung, T. R., Stewart, R. A., Sharma, A. K., \& Beal, C. D. (2014). Smart meters for enhanced water supply network modelling and infrastructure planning. Resources, Conservation and Recycling, 90, 34-50.

Hall, M. R., West, J., Sherman, B., Lane, J., \& de Haas, D. (2011). Long-Term Trends and Opportunities for Managing Regional Water Supply and Wastewater Greenhouse Gas Emissions. Environmental Science \& Technology, 45(12), 5434-5440.

House-Peters, L. A., \& Chang, H. (2011). Urban water demand modeling: review of concepts, methods, and organizing principles. Water Resources Research, 47(5).

Hussien, W. E. A., Memon, F. A., \& Savic, D. A. (2017). An integrated model to evaluate water-energyfood nexus at a household scale. Environmental Modelling \& Software, 93, 366-380.

Joachain, H., \& Klopfert, F. (2014). Smarter than metering? Coupling smart meters and complementary currencies to reinforce the motivation of households for energy savings. Ecological Economics, 105, 89-96.

Kabalci, Y. (2016). A survey on smart metering and smart grid communication. Renewable and Sustainable Energy Reviews, 57, 302-318.

Katsanevakis, M., Stewart, R. A., \& Lu, J. (2017). Aggregated applications and benefits of energy storage systems with application-specific control methods: A review. Renewable and Sustainable Energy Reviews, 75, 719-741.

Kaufmann, S., Künzel, K., \& Loock, M. (2013). Customer value of smart metering: Explorative evidence from a choice-based conjoint study in Switzerland. Energy Policy, 53, 229-239.

Kavousian, A., Rajagopal, R., \& Fischer, M. (2013). Determinants of residential electricity consumption: Using smart meter data to examine the effect of climate, building characteristics, appliance stock, and occupants' behavior. Energy, 55, 184-194.

Keelson, E., Boateng, K., \& Ghansah, I. (2014). A Smart Retrofitted Meter for Developing Countries. International Journal of Computer Applications, 90(5), 40-46.

Kenway, S. J., Binks, A., Lane, J., Lant, P. A., Lam, K. L., \& Simms, A. (2015). A systemic framework and analysis of urban water energy. Environmental Modelling and Software, 73, 272-285.

Kenway, S. J., Scheidegger, R., Larsen, T. A., Lant, P., \& Bader, H. P. (2013a). Water-related energy in households: a model designed to understand the current state and simulate possible measures. Energy and Buildings, 58, 378-389.

Kenway, S., McMahon, J., Elmer, V., Conrad, S., \& Rosenblum, J. (2013b). Managing water-related energy in future cities - a research and policy roadmap. Journal of Water and Climate Change, 4(3), 161-175. 
Khan, Z., Linares, P., García-González, J. (2017). Integrating water and energy models for policy driven applications. A review of contemporary work and recommendations for future developments. Renewable and Sustainable Energy Reviews, 67, 1123-1138.

Kiesling, L. (2016). Implications of Smart Grid Innovation for Organizational Models in Electricity Distribution. Smart Grid Handbook.

Kim, D., Park, K., Choi, G., \& Min, K. (2014). A study on the factors that affect the adoption of smart water grid. Journal of Computer Virology and Hacking Techniques, 10(2), 119-128.

Kim, H. W., Chan, H. C., \& Gupta, S. (2007). Value-based adoption of mobile internet: an empirical investigation. Decision Support Systems, 43(1), 111-126.

Kobus, C. B., Klaassen, E.A., Mugge, R., \& Schoormans, J. P. (2015). A real-life assessment on the effect of smart appliances for shifting households' electricity demand. Applied Energy, 147, 33543.

Kossieris, P., Kozanis, S., Hashmi, A., Katsiri, E., Vamvakeridou-Lyroudia, L.S., Farmani, R., Makropoulos, C. \& Savic, D. (2014). A web-based platform for water efficient households. Procedia Engineering, 89, 1128-1135.

Kroener, M., Allinger, K., Berger, M., Grether, E., Wieland, F., Heller, S., \& Woias, P. (2016). A water-powered Energy Harvesting system with Bluetooth Low Energy interface. Journal of Physics: Conference Series, 773, conference 1.

Kwac, J., Flora, J., \& Rajagopal, R. (2014). Household energy consumption segmentation using hourly data. IEEE Transactions on Smart Grid, 5(1), 420-430.

Lane, J. L., de Haas, D. W., \& Lant, P. A. (2015). The diverse environmental burden of city-scale urban water systems. Water Research, 81, 398-415.

Liu, A., Giurco, D., \& Mukheibir, P. (2015). Motivating metrics for household water-use feedback. Resources, Conservation and Recycling, 103, 29-46.

Liu, A., Giurco, D., Mukheibir, P. (2016). Urban water conservation through customised water and enduse information. Journal of Cleaner Production, 112(4), 3164-3175.

Liu, A., Turner, A., and White, S., 2017, Assessment of Future Water Efficiency Measures. Report prepared for City West Water, Yarra Valley Water, South East Water, MelbourneWater, Barwon Water and Department of Environment, Land, Water and Planning by the Institute for Sustainable Futures, University of Technology Sydney.

Lloret, J., Tomas, J., Canovas, A., \& Parra, L. (2016). An Integrated IoT Architecture for Smart Metering. IEEE Communications Magazine, 54(12), 50-57.

Loureiro, D., Alegre, H., Coelho, S., Martins, A., \& Mamade, A. (2014a). A new approach to improve water loss control using smart metering data. Water Science \& Technology Water Supply, 14(4), 618-625. 
Loureiro, D., Vieira, P., Makropoulos, C., Kossieris, P., Ribeiro, R., Barateiro, J., \& Katsiri, E. (2014b). Smart metering use cases to increase water and energy efficiency in water supply systems. Water Science \& Technology: Water Supply 14, 898-908.

Lundie, S., Peters, G. M., \& Beavis, P. C. (2004). Life Cycle Assessment for Sustainable Metropolitan Water Systems Planning. Environmental Science \& Technology, 38(13), 3465-3473.

Luthra, S., Kumar, S., Kharb, R., Ansari, M. F., \& Shimmi, S. (2014). Adoption of smart grid technologies: An analysis of interactions among barriers. Renewable and Sustainable Energy Reviews, 33, 554-565.

Ma, S., Yang, Y., Qian, Y., Sharif, H., \& Alahmad, M. (2016). Energy harvesting for wireless sensor networks: applications and challenges in smart grid. International Journal of Sensor Networks, 21(4), 226-241.

Makki, A. A., Stewart, R. A., Beal, C. D., \& Panuwatwanich, K. (2015). Novel bottom-up urban water demand forecasting model: Revealing the determinants, drivers and predictors of residential indoor end-use consumption. Resources, Conservation and Recycling, 95, 15-37.

Makropoulos, C., Kossieris, P., Kozanis, S., Katsiri, E. \& Vamvakeridou-Lyroudia, L. (2014). From smart meters to smart decisions: web-based support for the water efficient household. 11th International Conference on Hydroinformatics, HIC 2014, New York, USA, August 17-21, p. 1-8.

Marvin, S., Chappells, H., \& Guy, S. (1999). Pathways of smart metering development: shaping environmental innovation. Computers, Environment and Urban Systems, 23(2), 109-126.

McDaniel, P., \& McLaughlin, S. (2009). Security and privacy challenges in the smart grid. IEEE Security \& Privacy, 7(3), 75-77.

McHenry, M. P. (2013). Technical and governance considerations for advanced metering infrastructure/smart meters: technology, security, uncertainty, costs, benefits, and risks. Energy Policy, 59, 834-842.

Morsali, H., Shekarabi, S., Ardekani, K., Khayami, H., Fereidunian, A., Ghassemian M, Lesani, H. (2012). Smart plugs for building energy management systems. Proceedings of the 2nd Iranian Conference on Smart Grids (ICSG), p. 1-5, Tehran, Iran.

Mutchek, M., \& Williams, E. (2014). Moving towards sustainable and resilient smart water grids. Challenges, 5(1), 123-137.

Nadargi, A., \& Thirugnanam, M. (2016). Addressing identity and location privacy of things for indoor - case study on internet of everything's (IoE). Proceedings of the $3^{\text {rd }}$ International Symposium of Big Data and Cloud Computing Challenges (ISBCC - 16), p. 377-386, Tamil Nadu, India.

Nguyen, K. A., Stewart, R. A., \& Zhang, H. (2014). An autonomous and intelligent expert system for residential water end-use classification. Journal of Expert Systems with Application, 41(2), 342-356.

Nguyen, K. A., Stewart, R. A., Zhang, H., \& Jones, C. (2015). Intelligent autonomous system for residential water end use classification: Autoflow. Applied Soft Computing, 31, 118-131. 
Nguyen, K. A., Zhang, H., \& Stewart, R. A. (2013a). Intelligent pattern recognition model to automate the categorisation of residential water end-use events. Journal of Environmental Modelling and Software, 47, 108-127.

Nguyen, K. A., Zhang, H., \& Stewart, R. A. (2013b). Development of an intelligent model to categorise residential water end use events. Journal of Hydro-environment Research, 7(3), 182-201.

Nguyen, K. A., Zhang, H., \& Stewart, R. A. (2011). Application of dynamic time warping algorithm in prototype selection for the disaggregation of domestic water flow data into end use events. Proceeding of the 34th World Congress of the International Association for Hydro-Environment Engineering and Research, Brisbane, Australia.

Olivera, R., Duffya, A., \& Kilgallonba, I. (2016). Statistical models to infer gas end-use efficiency in individual dwellings using smart metered data. Sustainable Cities and Society, 23, 1-10.

Paetz, A. G., Dütschke, E., \& Fichtner, W. (2012). Smart homes as a means to sustainable energy consumption: A study of consumer perceptions. Journal of Consumer Policy, 35(1), 23-41.

Pallonetto, F, Oxizidis, S., Milano, F., and Finn, D. (2016). The effect of time-of-use tariffs on the demand response flexibility of an all-electric smart-grid-ready dwelling, Energy and Buildings 128(2016) 56-67.

Panuwatwanich, K., \& Stewart, R. A. (2012). Evaluating innovation diffusion readiness among architectural and engineering design firms: Empirical evidence from Australia. Automation in Construction, 27, 50-59.

Parikh, P. P., Kanabar, M. G., \& Sidhu, T. S. (2010). Opportunities and challenges of wireless communication technologies for smart grid applications. Power and Energy Society General Meeting: IEEE, pp. 1-7, Minneapolis, Minnesota, USA.

Parkhill, K., Demski, C., Butler, C., Spence, A., \& Pidgeon, N. (2013). Transforming the UK energy system: public values, attitudes and acceptability: synthesis report. London, UK.

Piga, D., Cominola, A., Giuliani, M., Castelletti, A., \& Rizzoli, A. E. (2016). Sparse optimization for automated energy end use disaggregation. IEEE Transactions on Control Systems Technology, 24(3), 1044-1051.

Pitì, A., Verticale, G., Rottondi, C., Capone, A., \& Lo Schiavo, L. (2017). The role of smart meters in enabling real-time energy services for households: The Italian case. Energies, 10(2), 199.

Proenca, L. C., Ghisi, E., Tavares, D. D., \& Coelho, G. M. (2011). Potential for electricity savings by reducing potable water consumption in a city scale. Resources, Conservation and Recycling, 55(11), 960-965.

Poullikkas, A., Kourtis, G., \& Hadjipaschalis, I. (2013). A review of net metering mechanism for electricity renewable energy sources. International Journal of Energy \& Environment, 4(6).

Puust, R., Kapelan, Z., Savic, D., \& Koppel, T. (2010). A review of methods for leakage management in pipe networks. Urban Water Journal, 7(1), 25-45. 
Raza, U., Kulkarni, P., \& Sooriyabandara, M. (2017). Low power wide area networks: An overview. IEEE Communications Surveys \& Tutorials, pp (99).

Retamal, M., Abeysuriya, K., Turner, A., \& White, S. (2008). Water Energy Nexus: Literature Review. Institute for Sustainable Futures, University of Technology, Sydney.

Rhodes, J. D., Upshaw, C. R., Harris, C. B., Meehan, C. M., Walling, D. A., Navrátil, P. A., Beck, A. L., Nagasawa, K., Fares, R. L., \& Cole, W. J. (2014). Experimental and data collection methods for a large-scale smart grid deployment: Methods and first results. Energy, 65, 462-471.

Ripka, P. (2010). Electric current sensors: a review. Measurement Science and Technology, 21(11), 112001.

Rizzoli, A. E. Castelleti, A. Cominola, A. Fraternali, P. Diniz dos Santos, A. Storni, B. WissmannAlves, R. Bertocchi, M. Novak, J.and Micheal, I. (2014). The SmartH2O project and the role of social computing in promoting efficient residential water use: a first analysis. (June 17, 2014). International Congress on Environmental Modelling and Software. Paper 19. http://scholarsarchive.byu.edu/iemssconference/2014/Stream-H/19

Rogers, W. and Carroll, P. (2016) Smart meter tariff design to minimise wholesale risk, Eletronic Notes in Discrete Mathematics 52, 21-28.

Rohracher, H. (2003). The role of users in the social shaping of environmental technologies. Innovation: the European Journal of Social Science Research, 16(2), 177-192.

Rottondi, C., Verticale, G., \& Capone, A. (2013). Privacy-preserving smart metering with multiple data Consumers. Computer Networks, 57(7), 1699-1713.

Savić, D., Vamvakeridou-Lyroudia, L. \& Kapelan, Z. (2014). Smart meters, smart water, smart societies: The iWIDGET project. Procedia Engineering 89, p. 1105-1112.

SGCC (2013). Smart Grid Economic and Environmental Benefits. A Review and Synthesis of Research on Smart Grid Benefits and Costs: Smart Grid consumer collaborative.

Siano, P. (2014). Demand response and smart grids-A survey. Renewable and Sustainable Energy Reviews, 30, 461-478.

Siems, R., \& Sahin, O. (2016). Energy intensity of residential rainwater tank systems: exploring the economic and environmental impacts. Journal of Cleaner Production, 113, 251-262.

Skopik, F., Ma, Z., Bleier, T., \& Grüneis, H. (2012). A survey on threats and vulnerabilities in smart metering infrastructures. International Journal of Smart Grid and Clean Energy, 1(1), 22-28.

Soldatos, J., Serrano, M., \& Hauswirth, M. (2012). Convergence of utility computing with the internetof-things. Proceedings of the Sixth International Conference on Innovative Mobile and Internet Services in Ubiquitous Computing (IMIS), pp. 874-879, Palermo, Italy.

Sønderlund, L., Smith, R., Hutton, J., Kapelan, Z., \& Savic, D. (2016). Effectiveness of smart meterbased consumption feedback in curbing household water use: knowns and unknowns. Journal of Water Resources Planning and Management, 142(12). 
Spang, E. S., \& Loge, F. J. (2015). A high-resolution approach to mapping energy flows through water infrastructure systems. Journal of Industrial Ecology, 19(4), 656-665.

Spang, E. S., Loge, F. J., Abernathy, W., Dove, D. R., Tseng, C., \& Williams, M. (2015). Implementing Consumption-Based Fixed Rates in Davis, Calif. American Water Works Association, 107(7).

Squartini, S., Liu, D., Piazza, F., Zhao, D., \& He, H. (2015) Computational Energy Management in Smart Grids. Neurocomputing, 170, 267-269.

Stewart, R. (2011). Verifying the end use potable water savings from contemporary residential water supply schemes. National Water Commission Waterlines Report No. 61, Australian Government, Canberra.

Stewart, R. A. (2008). A framework for the life cycle management of information technology projects: Project IT. International Journal of Project Management, 26(2), 203-212.

Stewart, R. A., Mohamed, S., \& Daet, R. (2002). Strategic implementation of IT/IS projects in construction: a case study. Automation in Construction, 11(6), 681-694.

Stewart, R., Giurco, D., \& Beal, C. (2013). Age of intelligent metering and big data: Hydroinformatics challenges and opportunities. Journal of the International Association for Hydro-environment Engineering and Research, 2, 107-110.

Stewart, R., Willis, R., Giurco, D., Panuwatwanich, K., \& Capati, G. (2010). Web-based knowledge management system: linking smart metering to the future of urban water planning. Australian Planner, 47(2), 66-74.

Surles, W., \& Henze, G. P. (2012). Evaluation of automatic priced based thermostat control for peak energy reduction under residential time-of-use utility tariffs. Energy and buildings, 49, 99-108.

Talebpour, M. R., Sahin, O., Siems, R., \& Stewart, R. A. (2014). Water and energy nexus of residential rainwater tanks at an end use level: Case of Australia. Energy and Buildings, 80, 195-207.

Taormina, R., Galelli, S., Tippenhauer, N. O., Salomons, E., \& Ostfeld, A. (2017). Characterizing cyber-physical attacks on water distribution systems. Journal of Water Resources Planning and Management, 143(5), 04017009.

Tayal, D. (2016). Disruptive forces on the electricity industry: A changing landscape for utilities. The Electricity Journal, 29(7), 13-17.

Tuballa, M. L., \& Abundo, M. L. (2016). A review of the development of Smart Grid technologies. Renewable and Sustainable Energy Reviews, 59, 710-725.

Turner, A., (2015) Integrated Resource Planning the Smart Metering Era, presentation at the IWA conference on Water Efficiency and Performance Assessment of Water Services, Cincinnati, Ohio, USA, April 20-24 2015

Turner, A., Willets, J., Fane, S., Giurco, D., Chong, J., Kazaglis, A., \& White S. (2010). Guide to demand management and integrated resource planning. Prepared by the Institute for Sustainable Futures, University of Technology Sydney for the National Water Commission and the Water Services Association of Australia, Inc. 
Turner, A., \& White, S., (2017). Urban water futures: Trends and potential disruptions. Report prepared by the Institute for Sustainable Futures, University of Technology Sydney, for the Water Services Association of Australia (WSAA).https://opus.lib.uts.edu.au/handle/10453/77441

van der Maaten, L., Hinton, G., 2008. Visualizing data using t-sne. Journal of Machine Learning Research 9, 2579-2605.

Vassileva, I., \& Campillo, J. (2014). Increasing energy efficiency in low-income households through targeting awareness and behavioral change. Renewable Energy, 67, 59-63.

VICGOV (2014). Quantifying Smart Meter RF EME Levels In Victorian Homes: Department Of Economic Development, Jobs, Transport And Resources. Victoria, Australia.

Vieira, A. S., Beal, C. D., \& Stewart, R. A. (2014a). Residential water heaters in Brisbane, Australia: Thinking beyond technology selection to enhance energy efficiency and level of service. Energy and Buildings, 82, 222-236.

Vieira, A. S., Beal, C. D., Ghisi, E., \& Stewart, R. A. (2014b). Energy intensity of rainwater harvesting systems: A review. Renewable and Sustainable Energy Reviews, 34, 225-242.

Villani, C., Benatti, S., Brunelli, D., \& Benini, L. (2016). A contactless three-phase autonomous power meter. IEEE SENSORS, Orlando, FL, USA, pp. 1-3.

Vojdani, A. (2008). Smart integration. IEEE Power and Energy Magazine, 6(6), 71-79.

Wang, S., Xue, X., \& Yan, C. (2014). Building power demand response methods toward smart grid. HVAC\&R Research, 20(6), 665-687.

WaterSmart, (2015). Participant Entry Survey. San Francisco, CA: WaterSmart Software.

Willis, R. M., Stewart, R. A., Giurco, D. P., Talebpour, M. R., \& Mousavinejad, A. (2013). End use water consumption in households: impact of socio-demographic factors and efficient devices. Journal of Cleaner Production, 60, 107-115.

Willis, R. M., Stewart, R. A., Panuwatwanich, K., Jones, S., \& Kyriakides, A. (2010). Alarming visual display monitors affecting shower end use water and energy conservation in Australian residential households. Resources, Conservation and Recycling, 54(12), 1117-1127.

Wilson, C., Hargreaves, T., \& Hauxwell-Baldwin, R. (2015). Smart homes and their users: a systematic analysis and key challenges. Personal and Ubiquitous Computing, 19(2), 463-476.

Yan, Y., Qian, Y., Sharif, H., \& Tipper, D. (2013). A survey on smart grid communication infrastructures: Motivations, requirements and challenges. IEEE Communications Surveys \& Tutorials, 15(1), 5-20.

Yaqoob, I., Hashem, I. A. T., Mehmood, Y., Gani, A., Mokhtar, S., \& Guizani, S. (2017). Enabling communication technologies for smart cities. IEEE Communications Magazine, 55(1), 112-120.

Zeifman, M., \& Roth, K. (2011). Nonintrusive appliance load monitoring: review and outlook. IEEE Transactions on Consumer Electronics, 57(1), 76-84.

Zhou, K., Fu, C., \& Yang, S. (2016). Big data driven smart energy management: From big data to big insights. Renewable and Sustainable Energy Reviews, 56, 215-225. 
Zhou, S., \& Brown, M. A. (2017). Smart meter deployment in Europe: A comparative case study on the impacts of national policy schemes. Journal of Cleaner Production, 144, 22-32.

Zoha, A., Gluhak, A., Imran, M. A., \& Rajasegarar, S. (2012). Non-intrusive load monitoring approaches for disaggregated energy sensing: A survey. Sensors, 12(12), 16838-16866. 